\title{
Inhibition of hexokinase-2 with targeted liposomal 3-bromopyruvate in an ovarian tumor spheroid model of aerobic glycolysis
}

This article was published in the following Dove Press journal:

International Journal of Nanomedicine

8 July 2015

Number of times this article has been viewed

\section{Srujan Kumar Gandham \\ Meghna Talekar \\ Amit Singh \\ Mansoor M Amiji}

Department of Pharmaceutical Sciences, School of Pharmacy, Northeastern University, Boston, MA, USA
Correspondence: Mansoor M Amiji Department of Pharmaceutical Sciences, School of Pharmacy, Northeastern University, 140 The Fenway Building, RI56, 360 Huntington Avenue, Boston, MA 02115, USA

$\mathrm{Tel}+16173733137$

Fax + I 6173738886

Email m.amiji@neu.edu
Background: The objective of this study was to evaluate the expression levels of glycolytic markers, especially hexokinase-2 (HK2), using a three-dimensional multicellular spheroid model of human ovarian adenocarcinoma (SKOV-3) cells and to develop an epidermal growth factor receptor-targeted liposomal formulation for improving inhibition of HK2 and the cytotoxicity of 3-bromopyruvate (3-BPA).

Methods: Multicellular SKOV-3 tumor spheroids were developed using the hanging drop method and expression levels of glycolytic markers were examined. Non-targeted and epidermal growth factor receptor-targeted liposomal formulations of 3-BPA were formulated and characterized. Permeability and cellular uptake of the liposomal formulations in three-dimensional SKOV-3 spheroids was evaluated using confocal microscopy. The cytotoxicity and HK2 inhibition potential of solution form of 3-BPA was compared to the corresponding liposomal formulation by using cell proliferation and HK2 enzymatic assays.

Results: SKOV-3 spheroids were reproducibly developed using the 96-well hanging drop method, with an average size of $900 \mu \mathrm{m}$ by day 5 . HK2 enzyme activity levels under hypoxic conditions were found to be higher than under normoxic conditions $(P<0.0001$, Student's $t$-test, unpaired and two-tailed). Liposomal formulations (both non-targeted and targeted) of 3-BPA showed a more potent inhibitory effect $(P<0.001$, Student's $t$-test, unpaired and two-tailed) at a dose of $50 \mu \mathrm{M}$ than the aqueous solution form at 3,6, and 24 hours post administration. Similarly, the cytotoxic activity 3 -BPA at various concentrations $(10 \mu \mathrm{M}-100 \mu \mathrm{M})$ showed that the liposomal formulations had an enhanced cytotoxic effect of $2-5$-fold $(P<0.0001$, Student's $t$-test, unpaired and two-tailed) when compared to the aqueous solution form for both $10 \mu \mathrm{M}$ and $25 \mu \mathrm{M}$ concentrations.

Conclusion: SKOV-3 spheroids developed by the hanging drop method can be used as a tumor aerobic glycolysis model for evaluation of therapies targeting the glycolytic pathway in cancer cells. Encapsulation of 3-BPA in a liposomal formulation improved permeability, HK2 inhibition, and cytotoxicity in the multicellular spheroid model.

Keywords: ovarian cancer, aerobic glycolysis, hexokinase-2, 3-bromopyruvate, epidermal growth factor receptor-targeted liposomes

\section{Introduction}

Ovarian cancer is the leading cause of death among the gynecological cancers, and caused an estimated 14,270 deaths in 2014 in the USA. ${ }^{1}$ The high mortality rate can be attributed to a lack of effective diagnostics for early detection of ovarian cancer. Thus, most cases are diagnosed at advanced stages, for which the 5-year survival rate is merely $27 \%$. $^{2}$ In advanced stages, intravenous chemotherapy is the standard treatment option. However, systemic chemotherapy, which involves use of a combination of cytotoxic 
drugs like carboplatin and paclitaxel, lacks specificity for cancer cells, resulting in toxic side effects, poor efficacy, and in some cases relapse of the disease. ${ }^{2}$ Thus, there exists a huge unmet medical need for development of efficacious therapies for ovarian cancer that can improve the survival rate of these patients without the burden of off-target toxicity.

Increased glycolytic activity due to the hypoxic microenvironment in many types of solid tumors endows cancer cells with selective advantages like enhanced proliferation, invasion, and metastasis. ${ }^{3}$ This observation has led to the development of therapeutic strategies such as use of small molecules for inhibition of glycolytic activity in cancer cells. ${ }^{4}$ In vitro studies for testing these drugs are generally performed using two-dimensional (2D) monolayer cell cultures of human or murine tumor cells. However, these monolayer models fail to recapitulate the complex three-dimensional (3D) architecture of human tumors in vivo. ${ }^{5}$ This leads to a difference in gene expression patterns and functional phenotypes in cells grown as 2D cultures when compared with those of in vivo tumors, and also to different efficacy of anticancer drugs. ${ }^{6,7}$ Hence, there is a need for better biologically relevant in vitro models to study the phenotypic profile of cancer cells and evaluate therapies targeting their biochemical pathways.

Spheroids are microscale cell clusters formed by selfassembly of cultured cells and are popular for investigating the mechanisms of disease and for screening therapies, including anticancer drugs. ${ }^{8}$ Cells growing in a 3D environment or spheroid behave differently from cells cultured in a $2 \mathrm{D}$ monolayer, because cells in 3D culture have more physiological cell-cell contact geometry, chemical gradients, mass transport, and mechanical properties. ${ }^{9}$ This leads to creation of heterogeneous cell subpopulations within the spheroid, with actively proliferating cells on the periphery and the quiescent hypoxic and necrotic cells in the inner regions. ${ }^{10}$ Since hypoxia is known to upregulate the expression of glycolytic markers at the mRNA and protein levels, the presence of this metabolic alteration in spheroids turns out to be critical when testing anticancer therapeutics. The efficacy of drugs that target glycolytic enzymes is significantly altered in spheroids as compared with $2 \mathrm{D}$ culture, and provides a more accurate prediction of the efficacy of the same drug when used in vivo. In the present study, we developed a spheroid model of ovarian cancer to demonstrate the versatility of this approach in elucidating the biology of the disease and evaluating therapeutic strategies using various assays and methods.

3-Bromopyruvate (3-BPA) is a small-molecule pyruvate mimetic and anticancer drug, and its biological activity is attributed to alkylation of free thiol groups on the cysteine residues of proteins. ${ }^{11}$ In vitro studies in hepatocellular carcinoma, human leukemia (HL-60) cells, and lymphoma (Raji) cells, as well as in vivo studies in liver and lung cancer models, have revealed the inhibitory effects of 3-BPA on the glycolytic pathway, which are mediated by covalent modification of hexokinase-2 (HK2), the first enzyme in glycolysis. HK2 is responsible for catalyzing the adenosine triphosphate (ATP)-dependent first step of the glycolysis pathway, leading to depletion of ATP and significant loss of cell viability. ${ }^{12-14} \mathrm{HK} 2$ was found to be overexpressed in various types of cancer cells, leading to accelerated glycolytic uptake, and expression of this particular phenotype distinguishes cancer cells from normal cells. ${ }^{15}$ Additionally, RNA interference-mediated HK2 silencing was studied in both in vitro and in vivo models of non-small cell lung cancer, and the results were promising in terms of inhibition of tumor growth. ${ }^{16}$ However, because of the highly reactive alkylating nature of 3-BPA, its inhibitory effect is not restricted to HK2 alone, and this is believed to be the reason for its toxic systemic effects. ${ }^{17}$

Liposomes are the oldest and most extensively studied nanotechnology-based drug delivery system to date, and have a unique ability to encapsulate both hydrophilic and hydrophobic drugs in their aqueous core and bilayer, respectively. ${ }^{18}$ Liposomes have been used clinically to treat cancer and have demonstrated the following advantages: preferential accumulation and higher drug concentration at tumor sites, but not limited to the enhanced permeation and retention effect; better intracellular uptake and stability; improved biodistribution and pharmacokinetic profile of the drug; and higher therapeutic efficacy and lower toxicity. ${ }^{19,20}$ The therapeutic agent 3-BPA is a small polar molecule and its efficacy may be limited by its ability to cross the cell membrane. ${ }^{21}$ To improve the therapeutic efficacy of 3-BPA, we designed a novel liposomal formulation with a lipid bilayer consisting of 1,2-distearoyl-sn-glycero3-phosphocholine (DSPC), cholesterol, and 1,3 distearoyl-snglycero-3-phosphoethanolamine- $N$-[methoxy(polyethylene glycol)-2000] (DSPE-MPEG-2000), which can efficiently encapsulate 3-BPA in its hydrophilic core. The lipid bilayer composition was optimized by using different ratios of DSPE, cholesterol, and DSPE-MPEG-2000 to form stable nanosized particles that can encapsulate 3-BPA, uptake of which is mediated by endocytosis, and thereby increasing the amount of drug that can cross the cell membrane. To further increase the uptake of this liposomal formulation, we designed liposomes targeting the epidermal growth factor 
receptor (EGFR), a transmembrane receptor tyrosine kinase consisting of an extracellular ligand-binding region and an intracellular protein kinase domain (EGFR), which would lead to highly specific interaction between the surface of the liposomes and cellular uptake through receptor-mediated endocytosis. ${ }^{22}$ EGFR was chosen for active targeting mainly because of the expression of high levels of EGFR in various tumors and the fact that blocking EGFR has antitumor effects. $^{23}$ To achieve this, we modified the surface of the liposomes with a 12-amino acid, EGFR-specific peptide known as GE11 (YHWYGYTPQNVI) which has a GGGG spacer and a terminal cysteine residue that can be conjugated to the liposome surface via a heterobifunctional polyethylene glycol chain with maleimide (MAL) functionality. ${ }^{24}$

In this study, we evaluated the permeability, inhibitory effects on HK2, and cytotoxic potential of EGFR-targeted and non-targeted liposomal formulations in human ovarian adenocarcinoma (SKOV-3) cells using a 3D tumor spheroid model expressing aerobic glycolysis markers.

\section{Materials and methods Materials}

3-BPA (molecular weight 166.96), cholesterol, trifluoroacetic acid, and acetonitrile (high-performance liquid chromatography [HPLC] grade) were purchased from Sigma-Aldrich (St Louis, MO, USA), and a C18-alkyl reverse phase bonded HPLC column (Zorbax Sb-Aq, $5 \mu \mathrm{m}, 150 \mathrm{~mm} \times 4.1 \mathrm{~mm}$ ) was purchased from Agilent Technologies (Santa Clara, CA, USA). DSPC and DSPE-MPEG-2000 (molecular weight 2,000) were obtained from Lipoid (Ludwigshafen, Germany), DSPE-PEG-MAL (molecular weight 2,000) was sourced from Laysan Bio Inc (Arab, AL, USA), and dextranrhodamine $\mathrm{B}$ (molecular weight $10 \mathrm{kDa}$ ) was purchased from Life Technologies (Grand Island, NY, USA). The hexokinase assay kit was obtained from Abcam (Cambridge, MA, USA), a Pierce bicinchoninic acid assay kit was purchased from Thermo Scientific (Rockford, IL, USA), and a CellTiter-Glo ${ }^{\circledR}$ luminescent cell viability assay kit was obtained from Promega Corporation (Madison, WI, USA).

\section{Cell culture and spheroid development}

The human ovarian adenocarcinoma (SKOV-3) cell line was obtained from the American Type Culture Collection (Manassas, VA, USA) and grown in Roswell Park Memorial Institute 1640 medium supplemented with $10 \%$ fetal bovine serum and $1 \%$ penicillin/streptomycin. Hypoxic conditions were induced by placing the cultured flasks in a modulator incubator chamber (Billups-Rothenberg Inc, Del Mar, CA, USA) and by initially flushing with hypoxic gas $\left(0.5 \% \mathrm{O}_{2}, 5 \% \mathrm{CO}_{2}\right.$, and nitrogen balanced gas) as per the manufacturer's conditions. This chamber was placed in the cell culture incubator to induce an hypoxic environment in the flasks. The hanging drop method was used to form the SKOV-3 spheroids. When the cells grown in flasks (T75 and T150) reached $80 \%$ confluency, they were detached by trypsinization $(0.05 \%$ trypsin ethylenediaminetetraacetic acid), after which the cells were pelleted down by centrifugation at 1,200 rpm for 10 minutes, and a cell suspension with a concentration of 1,000 cells $/ \mu \mathrm{L}$ was prepared by adding fresh medium to the pellet. Next, $40 \mu \mathrm{L}$ of this cell suspension was seeded into each well of Perfecta $3 \mathrm{D}^{\circledR}$ hanging drop plates obtained from 3D Biomatrix (Burton, MI, USA) to form hanging drops. The reservoir of the plate was filled with sterilized water to prevent drying of the hanging drops. For harvesting the spheroids, the 96-well spheroid plate was placed on top of a 96-well plate and the spheroids were released from the wells by dispensing $100 \mu \mathrm{L}$ of $1 \times$ phosphate-buffered saline $(\mathrm{PBS})$.

\section{RT-PCR analysis of glycolytic markers in 2D cell culture and 3D spheroids mRNA extraction}

mRNA was extracted using a Genejet ${ }^{\circledR}$ RNA purification kit (Thermo Scientific, Pittsburgh, PA). Total mRNA was extracted from cells obtained on days 3 and 5 from 2D cell culture under normoxic and hypoxic conditions as well as from spheroids and quantified using a NanoDrop 2000 spectrophotometer (Thermo Scientific, Wilmington, DE, USA). The extracted mRNA was stored at $-80^{\circ} \mathrm{C}$ until further use.

\section{RT-PCR analysis}

One microgram of total mRNA was extracted from each sample and reverse transcribed, and amplification of the glycolytic genes, glucose transporter 1 (GLUT1), pyruvate kinase isoform M2 (PKM2), lactate dehydrogenase A (LDHA), and HK2 was performed using the SuperScript ${ }^{\circledR}$ III one-step reverse transcriptase polymerase chain reaction (RT-PCR) system (Invitrogen, Life Technologies). Beta actin was used as the control housekeeping gene. The following primer sequences were used for amplification: forward 5'-GATCTTCATGAGGTAGTCAG-3' and reverse 5'-CGAGAAGATGACCCAGATCA-3' ( $\beta$-actin); forward 5'-AACTCTTCAGCCAGGGTCCAC-3' and reverse 5'-CACAGTGAAGATGATGAAGAC-3' (GLUT1); forward 5'-ATTATTTGAGGAACTCCGCCGCCT-3' and reverse 5'-ATTCCGGGTCACAGCAATGATGG-3' 
(PKM2); forward 5'-TGGAGTGGAATGAATGTTGC-3' and reverse 5'-ATAGCCCAGGATGTGTAGCC-3' (LDHA); and forward $5^{\prime}$-ATTGTCCAGTGCATCGCGGA-3' and reverse 5'-TTCGCTTGCCCCATTATC-3' (HK2).

RT-PCR was performed using a thermo cycler (T100, Bio-Rad, Hercules, CA, USA) for 40 cycles for all the genes under investigation. The amplified reaction products were visualized on $2 \%$ E-gels with $\mathrm{SYBR}^{\circledR}$ Safe (Invitrogen) after running for 20 minutes. The gels were imaged on a ChemiDoc ${ }^{\mathrm{TM}}$ XRS imaging system (Bio-Rad) and the intensity of the bands was quantified using ImageLab ${ }^{\text {TM }}$ software. Densitometric analysis was used to evaluate the mRNA expression levels of the glycolytic genes under different experimental conditions.

\section{Analysis of HK2 enzyme levels}

Baseline HK2 levels in the spheroids were determined using the Abcam HK assay and compared between the normoxic and hypoxic cell cultures. This assay measures enzyme levels in a given sample by the amount of glucose that is converted nicotinamide adenine dinucleotide, which gives an absorbance signal at $450 \mathrm{~nm}$. An equal amount $(10 \mu \mathrm{L})$ of cell lysate was added to the wells of a 96-well assay plate in triplicates to $40 \mu \mathrm{L}$ of assay kit components (D-glucose, ATP, $\mathrm{Mg}^{2+}$ ) and the absorbance was measured using a plate reader (Bio-Tek Synergy HT, Winooski, VT, USA) in kinetic mode, with a time interval of 20 minutes between each reading. The activity of HK2 in each sample was then obtained from the nicotinamide adenine dinucleotide standard curve. The total protein content in each cell lysate was measured by bicinchoninic acid assay (Thermo Scientific). The HK2 activity for each sample was normalized to the total protein content in the respective samples.

\section{HPLC analysis of 3-BPA}

A HPLC method was developed to assess the concentration of 3-BPA. A C18-alkyl reverse phase bonded column (Zorbax $\mathrm{Sb}-\mathrm{Aq}$ ) was used for the stationary phase. The mobile phase consisted of a 90/10 mixture of $0.1 \%$ trifluoroacetic acid in water $/ 0.1 \%$ trifluoroacetic acid in acetonitrile. An isocratic elution method with a flow rate of $0.75 \mathrm{~mL}$ per minute and a run time of 10 minutes was used to detect 3-BPA in all the samples. 3-BPA was detected by measuring the absorbance at $204 \mathrm{~nm}$ by the ultraviolet detector (Waters, Medford, MA, USA). A standard curve for 3-BPA was generated using drug concentrations of $5 \mu \mathrm{g} / \mathrm{mL}, 10 \mu \mathrm{g} / \mathrm{mL}, 20 \mu \mathrm{g} / \mathrm{mL}, 50 \mu \mathrm{g} / \mathrm{mL}$, $100 \mu \mathrm{g} / \mathrm{mL}, 500 \mu \mathrm{g} / \mathrm{mL}$, and $1 \mathrm{mg} / \mathrm{mL}$, and blank solvents for 3-BPA were prepared in triplicate.

\section{Preparation and characterization of 3-BPA-encapsulated control and EGFR- targeted liposomes}

Control and EGFR-targeted liposomal formulations

A thin film hydration method was used to synthesize the liposomes. The lipid bilayer consisted of DSPC, cholesterol, and DSPE-MPEG-2000 at a molar ratio of 4:2:0.3 and the total weight of the lipids was $30 \mathrm{mg}$. The lipids were initially dissolved in $2 \mathrm{~mL}$ of chloroform in a round-bottomed flask, after which the solvent was evaporated using a rotary evaporator (RV 10, IKA, Wilmington, NC, USA) to yield a thin lipid film at the base of the flask. The lipid film was subjected to overnight vacuum drying to remove any residual organic solvent. The lipid film was then hydrated by adding PBS (pH 7) and 3-BPA (10 mg/mL; pH 1) dropwise to the round-bottomed flask while keeping it on hot plate stirrer maintained at $55^{\circ} \mathrm{C}-60^{\circ} \mathrm{C}$ to allow diffusion of 3-BPA molecules into the aqueous core of the liposomes. The resulting colloidal dispersion was transferred to a $20 \mathrm{~mL}$ scintillation vial and subjected to ultrasonication (Vibra Cell ${ }^{\circledR}$, Sonics Inc, Newtown, CT, USA) for 10 minutes with an amplitude of $21 \%$ and a pulse frequency of 1 second. Free 3 -BPA in the liposomal formulation was removed by size exclusion chromatography (Sephadex G-25 column, GE, Fairfield, CT, USA). Blank liposomes were prepared following the same procedure except for the hydration step, where deionized water was used instead of 3-BPA solution.

For the EGFR-targeted liposomes, DSPE-PEG-MAL was conjugated to the cysteine residues of the GE11 peptide in a 1:1 molar ratio while mixing under nitrogen gas in the presence of HEPES buffer $(\mathrm{pH} 7.4)$ at $4^{\circ} \mathrm{C}$, and the reaction was allowed to run overnight. Excess peptide was dialyzed out using a $2 \mathrm{kDa}$ dialysis cassette. The purified sample was then lyophilized and characterized by nuclear magnetic resonance to confirm the conjugation reaction. The peptidemodified DSPE-PEG-MAL was then used to prepare both the 3-BPA-encapsulated and blank EGFR-targeted liposomes, with the peptide-modified DSPE-PEG-MAL and DSPEMPEG-2000 added in a ratio of 1:1. The theoretical percentage amount of EGFR/GE11 peptide (molecular weight $1,872.1 \mathrm{~g} / \mathrm{mole}$ ) in the targeted liposomes in terms of mole ratio was 0.15 and its composition in moles was $2.3 \%$, with the other components being DSPC, cholesterol, and DSPEPEG (Figure S1).

Measurement of particle size and zeta potential

The particle size of the blank liposomes, non-targeted liposomes encapsulating 3-BPA, and EGFR-targeted liposomes 
encapsulating 3-BPA was measured using a Nano Zetasizer ${ }^{\circledR} 90$ (Malvern, Westborough, MA, USA). To measure the size of the particles, $10 \mu \mathrm{L}$ each of the blank liposomes and the liposomal formulations encapsulating 3-BPA were diluted with $990 \mu \mathrm{L}$ of deionized water and the temperature was maintained at $25^{\circ} \mathrm{C}$. The mean liposome diameter polydispersity index was reported for three batches of liposomes. The same dispersion was transferred to a cuvette for measurement of the zeta potential.

\section{Transmission electron microscopy}

A transmission electron microscope (TEM) was used to characterize and evaluate the surface morphology of the blank liposomes and the liposomes encapsulating 3-BPA. Uranyl acetate $(1.5 \%)$ was used as a negative staining method to visualize the liposomes. Next, $10 \mu \mathrm{L}$ each of the different liposomal formulations were diluted with $990 \mu \mathrm{L}$ of deionized water, and $10 \mu \mathrm{L}$ of each dispersion was placed on a carbon-over-pioloform-coated copper grid for 1 minute, after which $10 \mu \mathrm{L}$ of negative stain was used for 30 seconds. Excess sample and stain was blotted with Whatman filter paper, and the copper grid was air-dried for imaging using TEM (JEM-1000, JEOL, Tokyo, Japan) at 10,000× and 40,000× magnification.

\section{Encapsulation efficiency of liposomal 3-BPA}

The encapsulation efficiency (percent EE) of the drug-loaded liposomes was determined by HPLC. One $\mathrm{mL}$ of liposomes was disrupted with chloroform, vortexed vigorously for 10 minutes, and then centrifuged at 10,000 rpm for $10 \mathrm{~min}$ utes at $4^{\circ} \mathrm{C}$. The supernatant was then spiked with a known concentration of 3-BPA and analyzed by HPLC. Percent EE was calculated using the following formula:

$$
\% \mathrm{EE}=\left(\mathrm{C}_{\text {Liposomes }} / \mathrm{C}_{\text {Total }}\right) * 100
$$

where $\mathrm{C}_{\text {Liposomes }}$ is the concentration of 3-BPA in the liposomes as determined by HPLC for 3 -BPA and $\mathrm{C}_{\text {Total }}$ is the concentration used for loading the liposomes $* 100$.

\section{Confocal microscopy analysis of liposomal uptake and permeability in SKOV-3 2D culture and 3D spheroids}

For evaluation of cell uptake and permeability of the liposomes in SKOV-3 cells, liposomes were prepared in a manner similar to that used to prepare the liposomes encapsulating 3-BPA, but instead of 3-BPA, hydrophilic dextran-rhodamine B dye was encapsulated into the core of the liposomes using the method described in earlier sections. SKOV-3 spheroids were prepared by the previously described hanging drop method and harvested on day 5 into 96-well plates containing a known concentration of the dye encapsulated in liposomes and the solution form of the dye in separate wells of the plate. The spheroids were incubated for 30 minutes, 1 hour, or 2 hours in the presence of both forms of the dye. After this step, the spheroids were washed to remove excess dye and imaged by confocal microscopy (LSM 700 ${ }^{\circledR}$, Zeiss, Thornwood, NY, USA) using Z-stacking to check for permeability of the dye within the spheroids. Similarly, for the uptake studies, SKOV-3 cells were grown in 4-well chamber slides and treated with targeted and non-targeted liposomes encapsulating dextran-rhodamine for the same time periods. The cells were then washed with ice-cold PBS, fixed, stained with 4,6-diamidino-2-phenylindole (Millipore, Billerica, MA, USA), and imaged by confocal microscopy.

\section{Inhibition of HK2 activity in SKOV-3 cells and spheroids by control and targeted liposomal 3-BPA formulations}

To evaluate inhibition of HK2 using the non-targeted and targeted liposomal formulations of 3-BPA, SKOV-3 spheroids grown for 5 days were harvested and incubated with the three different formulations of 3-BPA (ie, the solution form, the non-targeted liposomes encapsulating 3-BPA, and EGFR-targeted liposomes encapsulating 3-BPA) loaded with $25 \mu \mathrm{M}, 50 \mu \mathrm{M}$, and $100 \mu \mathrm{M}$ concentrations of the drug, and HK2 expression was assessed using the HK assay (described earlier) following incubation for 3, 6, and 24 hours.

\section{Cytotoxicity of 3-BPA administered in control and targeted liposomal formulations}

The cytotoxic potential of the targeted and non-targeted liposomal formulations in SKOV-3 cells grown under normoxic and hypoxic conditions and in spheroids was evaluated using the CellTiter-Glo luminescent cell viability assay (Promega Corporation). Briefly, SKOV-3 cells grown under normoxic and hypoxic conditions were plated in 96-well plates at a seeding density of 3,000 cells/well incubated with three different formulations of 3-BPA (ie, the solution form, the non-targeted liposomes encapsulating 3-BPA, and EGFRtargeted liposomes encapsulating 3-BPA), loaded at doses of $10 \mu \mathrm{M}, 25 \mu \mathrm{M}, 50 \mu \mathrm{M}$, or $100 \mu \mathrm{M}$. Similarly, on day 5 , the spheroids were harvested into a 96-well plate using PBS and treated with the liposomes in a manner similar to that used for the normoxic cells. Blank liposomes and 
untreated cells in Roswell Park Memorial Institute 1640 medium supplemented with fetal bovine serum were used as controls for the experiment. After treatment for 3 or 24 hours, the surrounding medium was aspirated and the cells were washed twice with PBS. The CellTiter-Glo reagent was then added to all treatment and control groups, and the plates were shaken for 5 minutes. The luminescence of the product was measured using a plate reader. The luminescence intensity measures cellular ATP content, or cell viability, and is described relative to the intensity observed in untreated (healthy) cells as a control.

\section{Results}

\section{Development of tumor spheroids}

The hanging drop method enabled successful formation of compact SKOV-3 cell spheroids (Figure 1). Microscopic evaluation indicated formation of more compact spheroids on day 6 when compared with day 4 . There was some morphological variation in the spheroids, but they had an average size of $800 \pm 150 \mu \mathrm{m}$.

\section{RT-PCR analysis of glycolytic markers in 2D cell culture and 3D spheroids}

Agarose gel images (Figure S2) indicate that SKOV-3 cells expressed glycolytic markers, ie, GLUT1, HK2, PKM2, and
LDHA, when grown under all three conditions, ie, normoxia, hypoxia, and in spheroids. The intensity levels of the bands also indicate that GLUT1, which encodes for the glucose transporter, was highly expressed in all three conditions, ie, normoxia, hypoxia, and in spheroids, when compared with the other glycolytic markers, and this is in agreement with the high glucose uptake in cancer cells. Densitometric analysis (Figure $\mathrm{S} 3 ; \mathrm{n}=3$ ) showed no significant difference in expression of the glycolytic markers, which were (normalized to $\beta$-actin for each gene) in hypoxic conditions when compared with normoxic conditions at the mRNA level. Note that densitometric analysis could not be performed for HK2 because of the lower intensity levels (ie, faint bands in the gel for HK2).

\section{Analysis of HK2 enzyme levels}

Analysis of normalized hexokinase levels (Figure 2) indicated upregulation (a 2-3-fold increase, $P<0.0001$, one-way analysis of variance followed by Dunnett's multiple comparisons $t$-test) of enzyme levels of HK2 in SKOV-3 cells grown under hypoxic conditions (both hypoxia and in spheroids) on day 5 when compared with cells grown under normal oxygen conditions. This is in agreement with the hypothesis that hypoxia in the tumor microenvironment induces aerobic glycolysis and is the driving force for elevated glycolytic activity
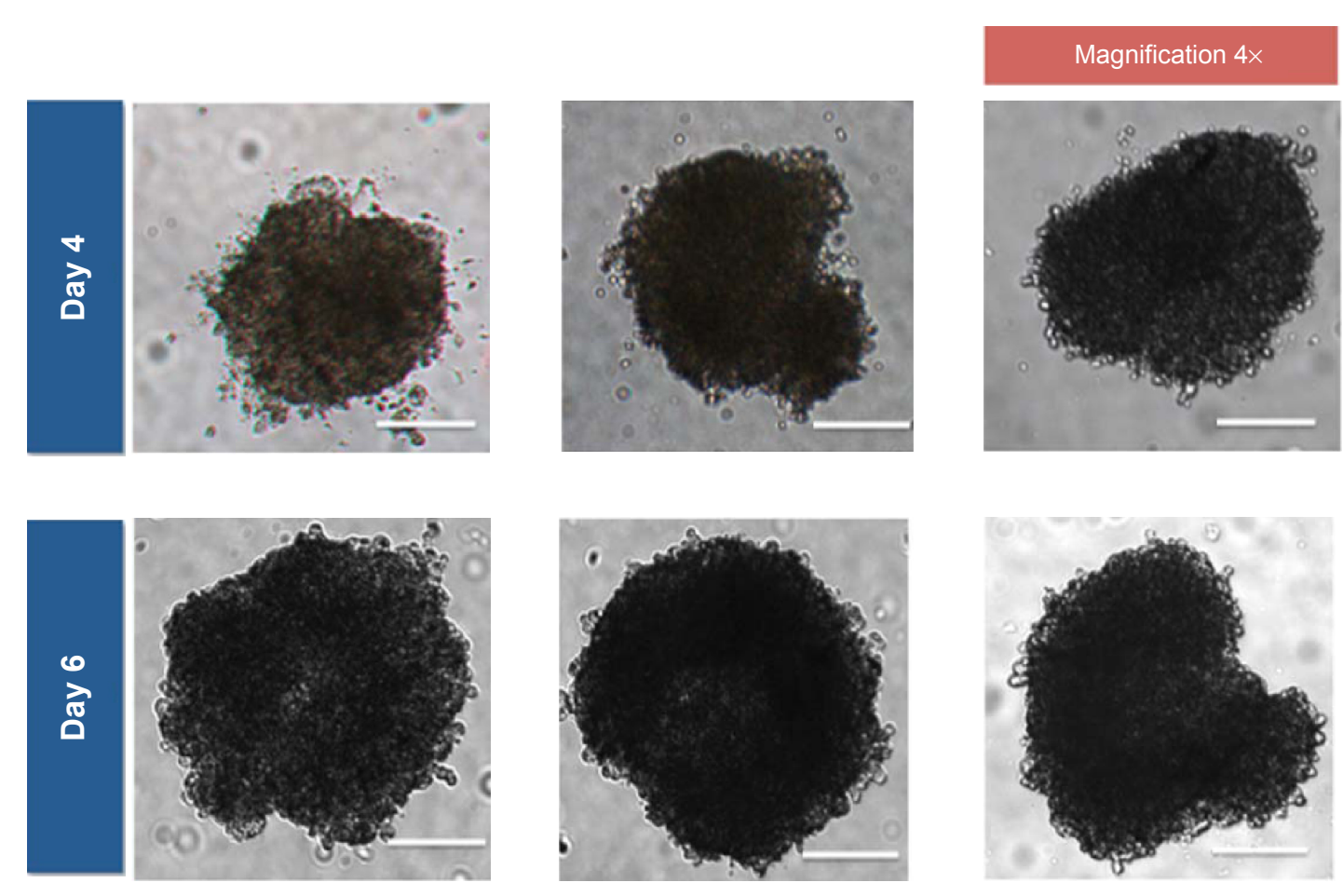

Figure I Microscopic images of human ovarian adenocarcinoma (SKOV-3) multicellular spheroids formed by the hanging drop method and harvested on days 4 and 6 after initiation of spheroid formation.

Notes: The scale bars correspond to $200 \mu \mathrm{m}$. 
Day 3 HK2 assay

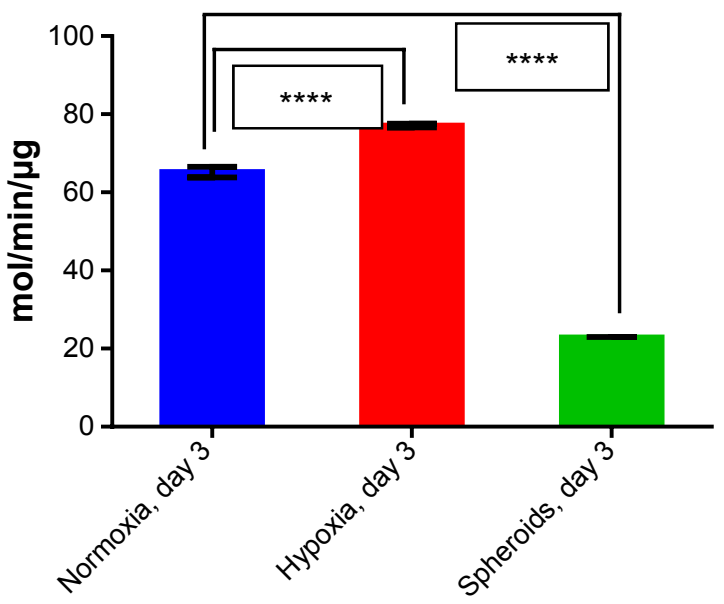

Day 5 HK2 assay

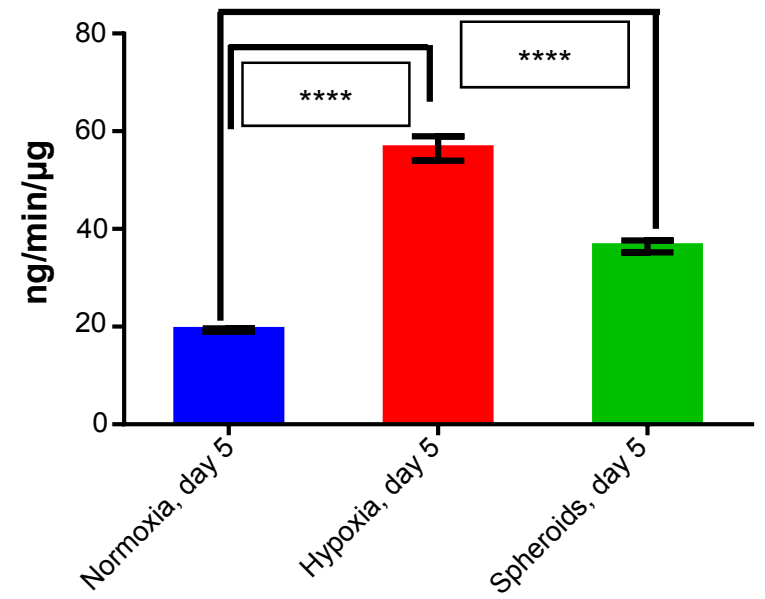

Figure 2 HK-2 activity levels in a two-dimensional ovarian cancer cell (SKOV-3) culture and in three-dimensional spheroids formed on days 3 and 5 normalized to total protein content in each sample.

Notes: The values are reported as the mean \pm standard deviation calculated for $n=3$. $* * * * P<0.000$ I, one-way analysis of variance followed by Dunnett's multiple comparisons $t$-test (a day 3 normoxia sample was used as the control).

Abbreviations: HK2, hexokinase-2; min, minutes.

or higher levels of glycolytic enzymes like HK2. In contrast with the day 5 spheroid samples, the HK2 activity in day 3 spheroid samples was lower than that observed under hypoxic conditions. This difference in HK2 expression is attributable to the time lag required for the buildup of a hypoxic microenvironment following formation of spheroid.

\section{Preparation and characterization of control liposomes and EGFR-targeted}

\section{3-BPA-loaded liposomes}

\section{Particle size, zeta potential, and EE}

The average particle size (hydrodynamic diameter), zeta potential, and EE of both the blank and 3-BPA-loaded liposomes are shown in Table 1. The mean particle size of the blank liposomes and 3-BPA-loaded liposomes were found to be 140-190 nm, with a narrow size distribution (polydispersity index). The zeta potential was found to be negative, ranging between -15 and $-20 \mathrm{mV}$ for both the blank and 3-BPA-loaded liposomes, and this can be explained by the outer surface coating of the particles by polyethylene glycol. The drug content of the liposomal formulations (percent EE) was determined by HPLC and found to be $10.2 \% \pm 0.5 \%$, and this can be explained by the highly polar nature of 3-BPA, which makes it restricted to the hydrophilic core of the liposomes.

\section{Surface conjugation of EGFR-targeting peptide}

${ }^{1} \mathrm{H}$-nuclear magnetic resonance analysis of EGFR-conjugated DSPE-PEG-MAL (Figure 3) revealed that the conjugate had peaks corresponding to hydrogens from the methyl $\left(\mathrm{CH}_{3}\right)$ groups of the lipid chain and hydrogens of the methoxy group $\left(\mathrm{OCH}_{2}\right)$ from the polyethylene glycol groups, but the peaks corresponding to the hydrogen of the $\mathrm{C}=\mathrm{C}$ of the MAL ring disappeared, indicating conjugation between the sulfhydryl group of the EGFR peptide and the MAL ring of the DSPE-PEG-MAL.

\section{TEM images of liposomes}

The TEM images (Figure S4) revealed that the surface morphology of the 3-BPA-loaded liposomes was spherical, with a lipid bilayer stained by uranyl acetate solution. The size distribution of the liposomes observed using TEM was in agreement with the particle size data obtained from the Zetasizer.

Table I Average liposome particle size, zeta potential, and percent EE of 3-BPA-loaded liposomes

\begin{tabular}{lllll}
\hline Formulation & Size $(\mathbf{n m})$ & PDI & Zeta potential (mV) & Percent EE \\
\hline Blank (non-targeted) liposomes & $144.13 \pm 14.21$ & 0.080 & $15.75 \pm 2.3$ & NA \\
Non-targeted 3-BPA loaded liposomes & $171.66 \pm 13.52$ & 0.0923 & $17.2 \pm 0.6$ & $10.46 \pm 0.52$ \\
Blank EGFR-targeted liposomes & $169.23 \pm 15.31$ & 0.109 & $19.7 \pm 0.4$ & $\mathrm{NA}$ \\
EGFR targeted 3-BPA-loaded liposomes & $184.71 \pm 13.42$ & 0.143 & $16.2 \pm 0.9$ & $10.02 \pm 0.27$ \\
\hline
\end{tabular}

Note: All values are reported as the mean \pm the standard deviation $(n=3)$.

Abbreviations: 3-BPA, 3-bromopyruvate; EE, encapsulation efficiency; EGFR, epidermal growth factor receptor; NA, not applicable; PDI, polydispersity index. 


\section{NMR analysis DSPE-PEG-MAL-EGFR}

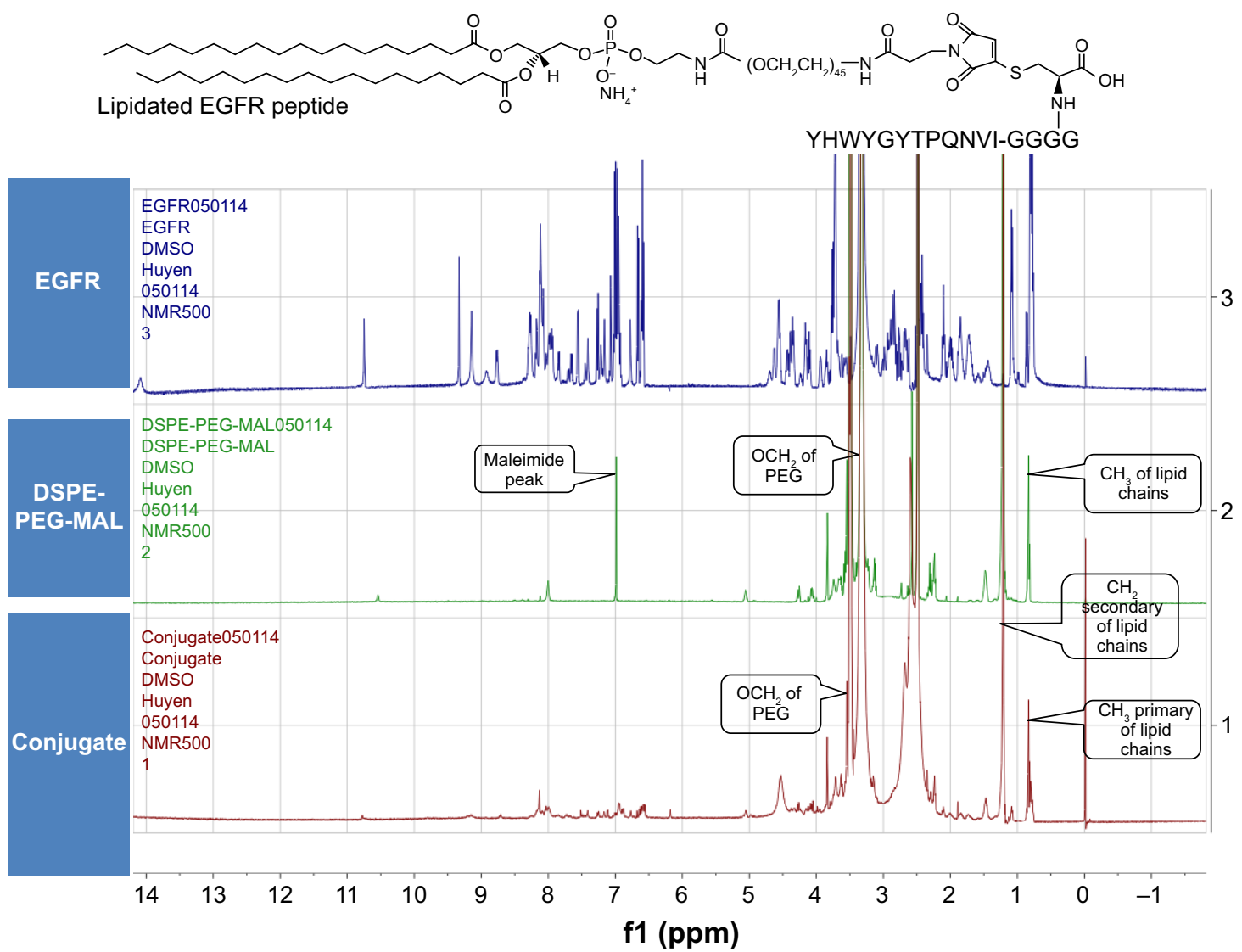

Figure 3 Comparison of ' $\mathrm{H}$-nuclear magnetic resonance spectra for EGFR-targeting peptide, DSPE-PEG-MAL, and the conjugated product.

Abbreviations: DMSO, dimethyl sulfoxide; DSPE, I,2-distearoyl-sn-glycero-3-phosphoethanolamine; EGFR, epidermal growth factor receptor; MAL, maleimide; NMR, nuclear magnetic resonance; PEG, polyethylene glycol.

\section{Cell uptake and permeability of SKOV-3 spheroids on confocal microscopy}

Figure 4 shows the permeability of the rhodamine-dextran fluorescent dye in solution versus the rhodamine-dextran encapsulated in the non-targeted and targeted liposomes. Comparison reveals an increased fluorescence signal from the rhodamine-dextran encapsulated within the liposomal formulations compared with the rhodamine-dextran in solution when the spheroids were scanned at specific depths, especially for lower depths at time points of 30 minutes (Figure 4A) and 1 hour (Figure 4B) for day 5 spheroid samples. This demonstrates the improved efficiency of the liposomal formulations in terms of penetrating the 3D tumor model and delivering the drug when compared with the drug given in solution. However, a significant difference was not observed between the targeted and non-targeted formulations.

Similarly, Figure 5A-C, which shows confocal microscopic images of cell uptake, reveals the signal from the rhodamine channel, indicating uptake of the liposomes by SKOV-3 cells and release of encapsulated rhodamine-dextran from the formulations. However, there was little difference between targeted and non-targeted liposomes in terms of uptake and signal.

\section{Inhibition of HK2 activity in spheroids with 3-BPA using control and EGFR- targeted liposomal formulations}

The hexokinase inhibition study (Figure 6) shows that the liposomal formulations (both non-targeted and targeted) had a higher or more potent inhibitory effect when compared with the 3-BPA solution at all three time points $(3,6$, and 24 hours) for the $50 \mu \mathrm{M}$ concentration of 3-BPA $(P<0.01$, Student's $t$-test, unpaired and two-tailed), while the $25 \mu \mathrm{M}$ concentration of 3-BPA seemed to show an inhibitory effect only at 6 and 24 hours. The $100 \mu \mathrm{M}$ concentration of 3-BPA had a cytotoxic effect, and hexokinase levels were found to below the detection limits of the assay. 

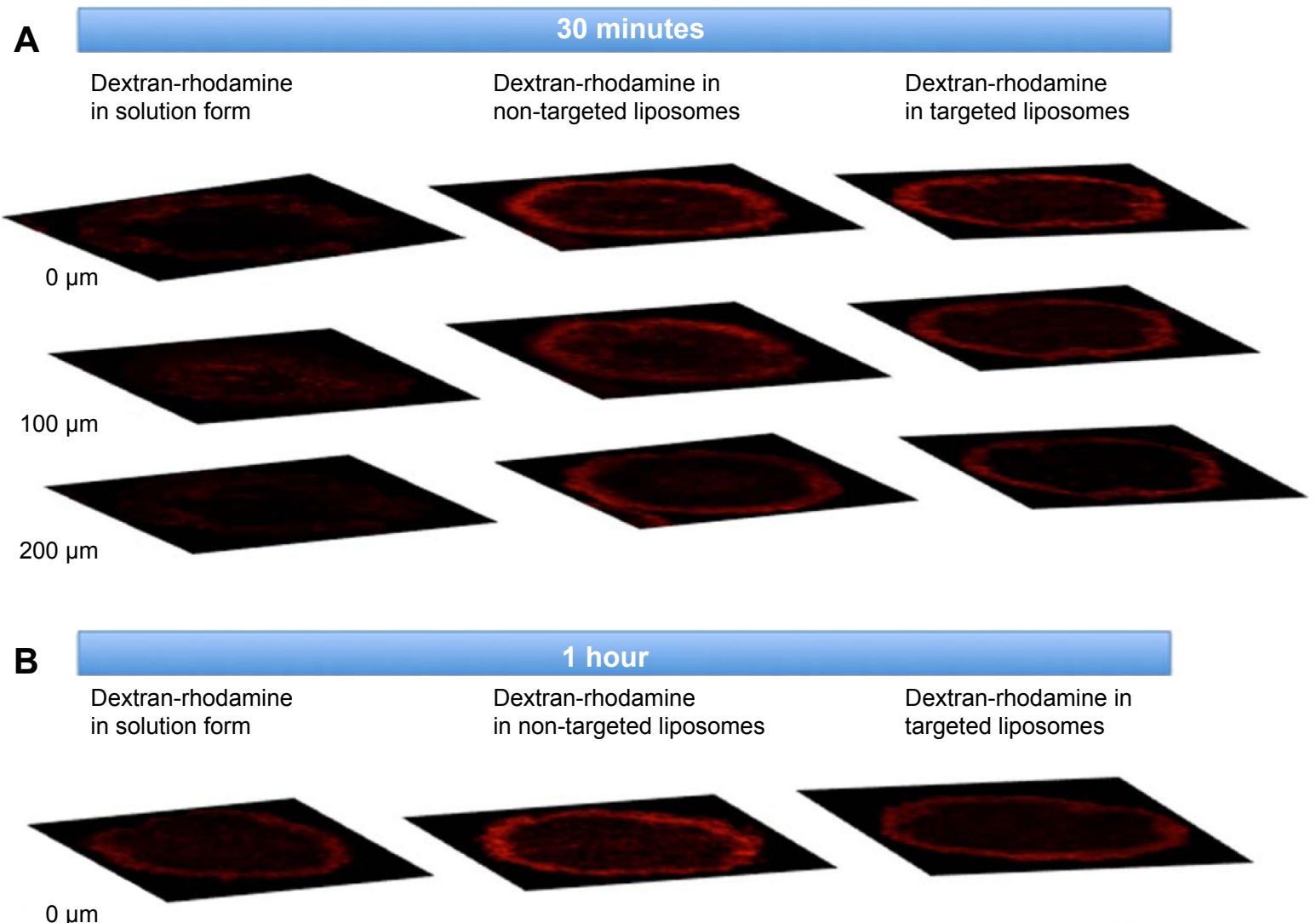

$0 \mu \mathrm{m}$
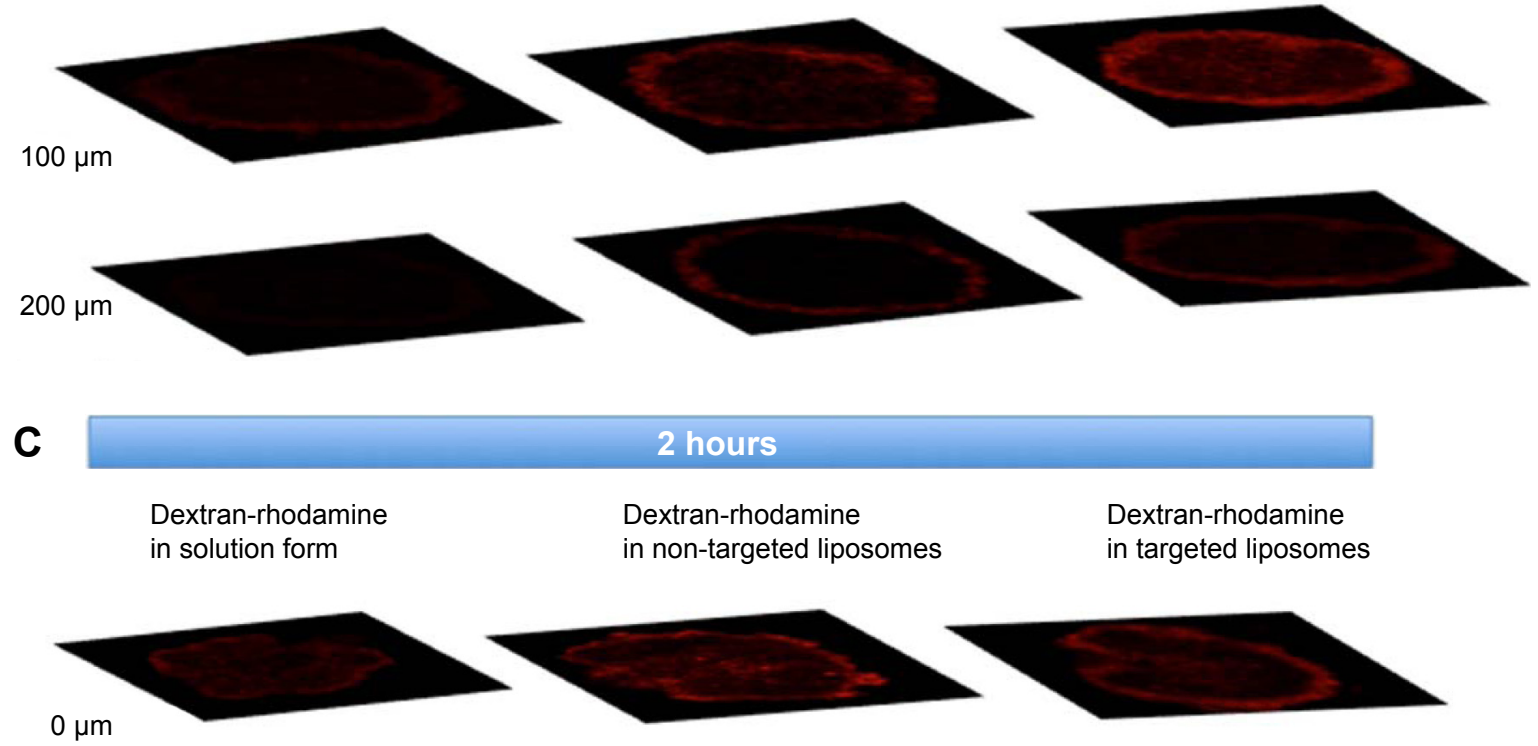

$0 \mu \mathrm{m}$
in solution form
in non-targeted liposomes
in targeted liposomes

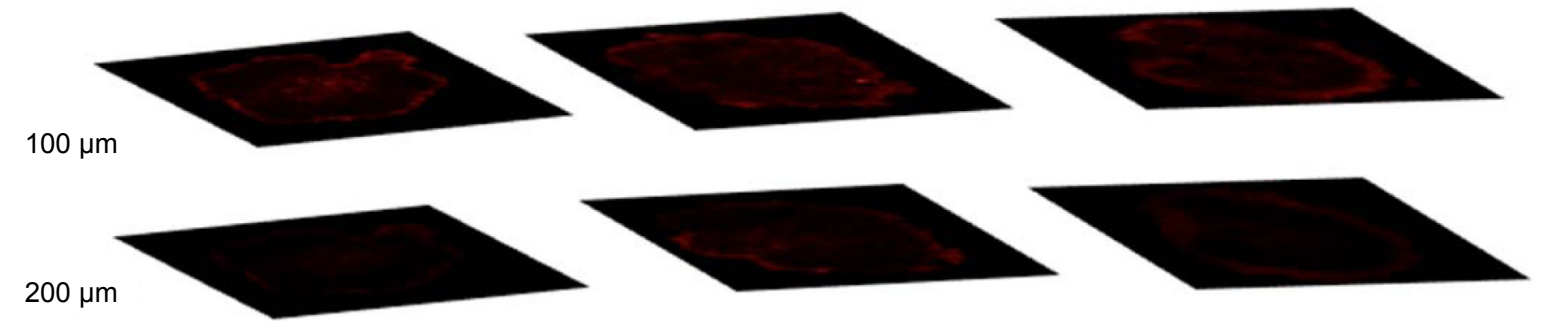

Figure 4 Qualitative evaluation of the permeability of non-targeted and targeted liposomal formulations in SKOV-3 spheroids.

Notes: Confocal microscopy Z-stacking images comparing permeability of rhodamine-conjugated dextran (molecular weight $10 \mathrm{kDa}$ ) in aqueous solution and encapsulated liposomal form at various depths from the top of the spheroid, ie, $0 \mu \mathrm{m}, 100 \mu \mathrm{m}$, and $200 \mu \mathrm{m}$ at (A) 30 minutes, (B) I hour, and (C) 2 hours after administration of the fluorescent dye. 
A

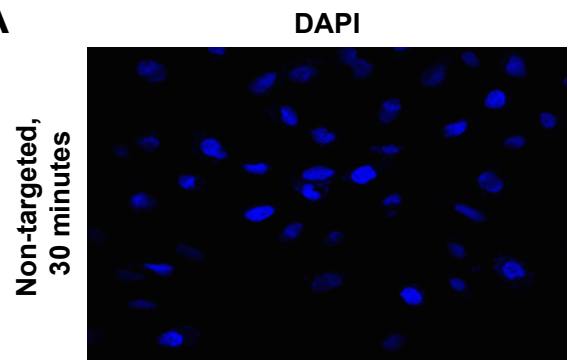

DAPI

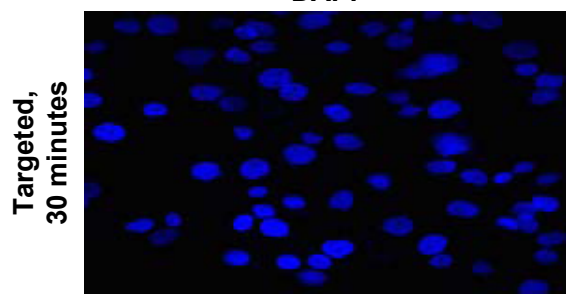

B

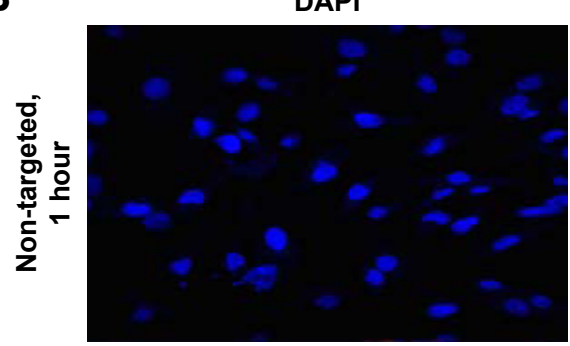

DAPI

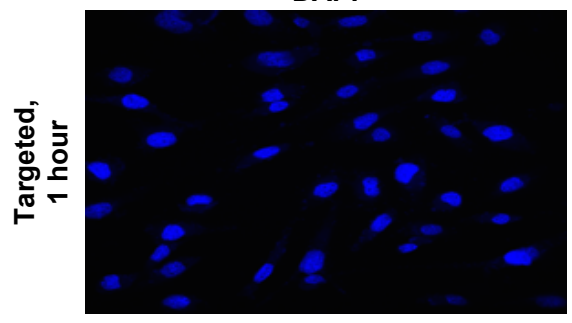

C

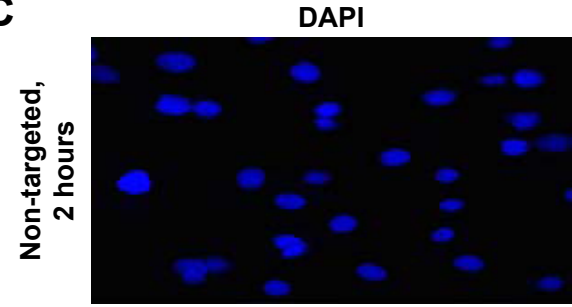

DAPI

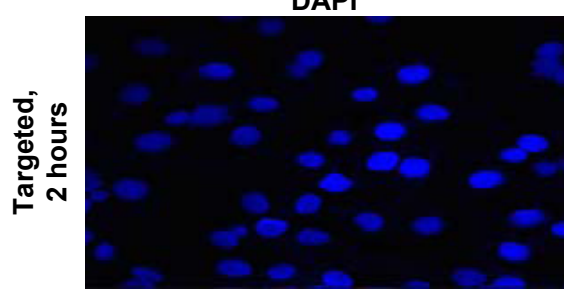

Rhodamine

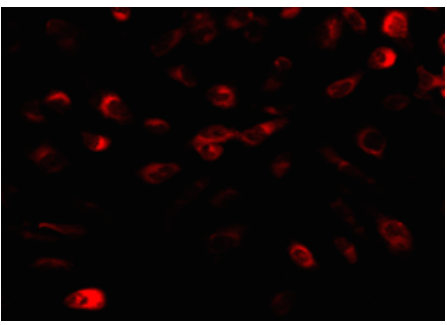

Rhodamine

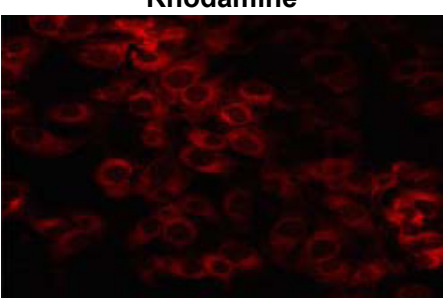

Rhodamine

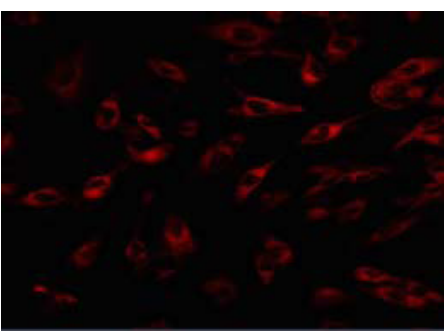

Rhodamine

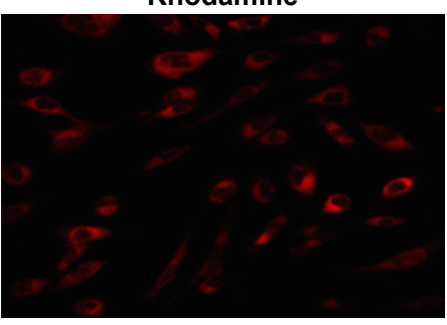

Rhodamine

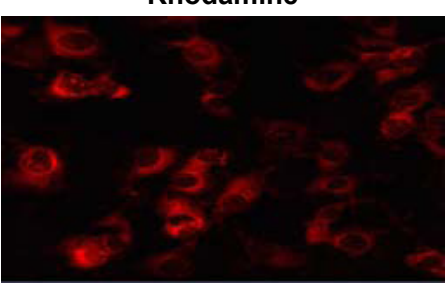

Rhodamine

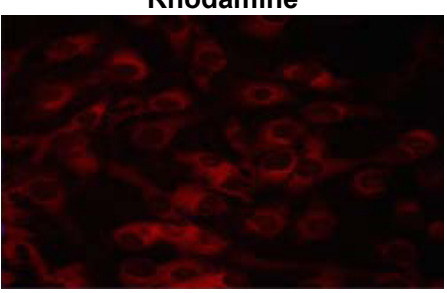

Merge

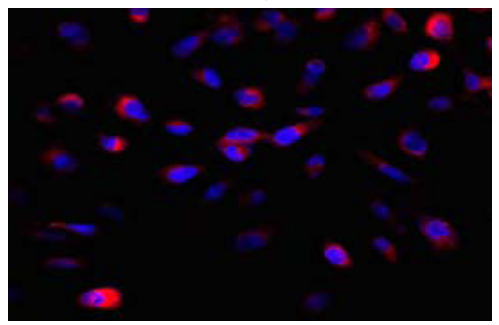

Merge

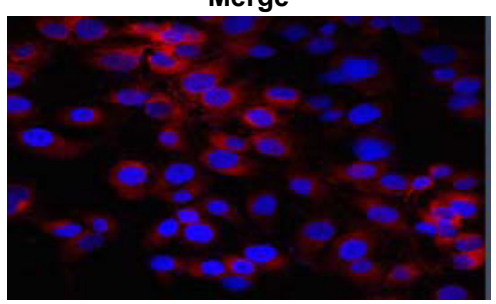

Merge

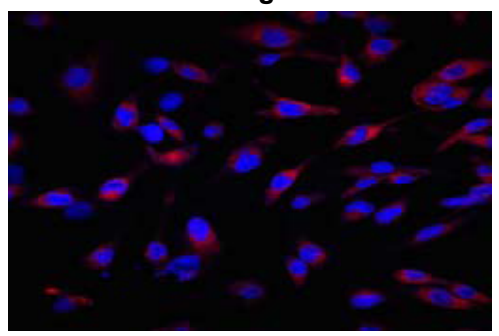

Merge

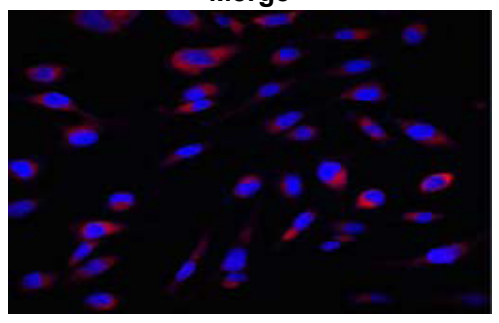

Merge

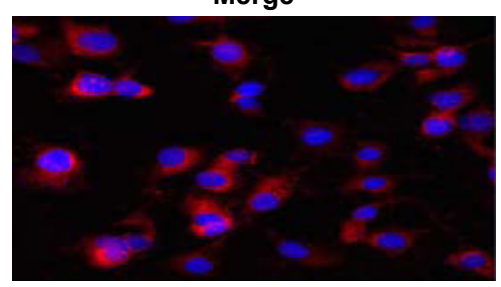

Merge

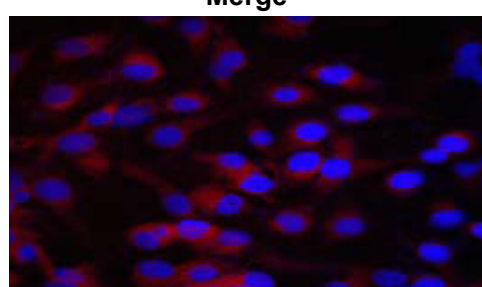

Figure 5 Uptake studies in SKOV-3 cells using non-targeted and targeted liposomal formulations.

Notes: Confocal microscopy images showing uptake of liposomal formulations by SKOV-3 cells incubated for various time periods, ie, (A) 30 minutes, (B) I hour, and (C) 2 hours after treatment. The cell nucleus was stained with DAPI (blue) and the liposomes were encapsulated with rhodamine-conjugated dextran (molecular weight $10 \mathrm{kDa}$, red). All images were captured using a $60 \times$ objective lens.

Abbreviation: DAPI, 4,6-diamidino-2-phenylindole. 
HK2 activity, spheroids, 3-hour sample

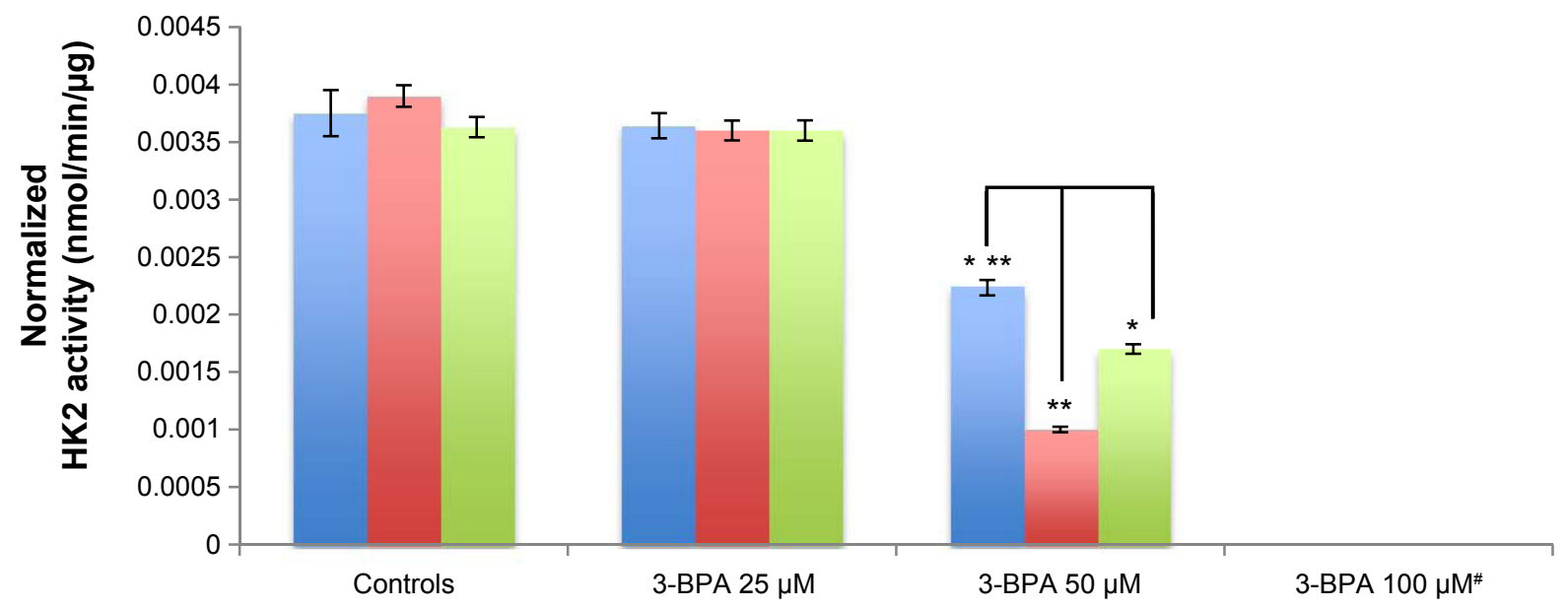

HK2 activity, spheroids, 6-hour sample
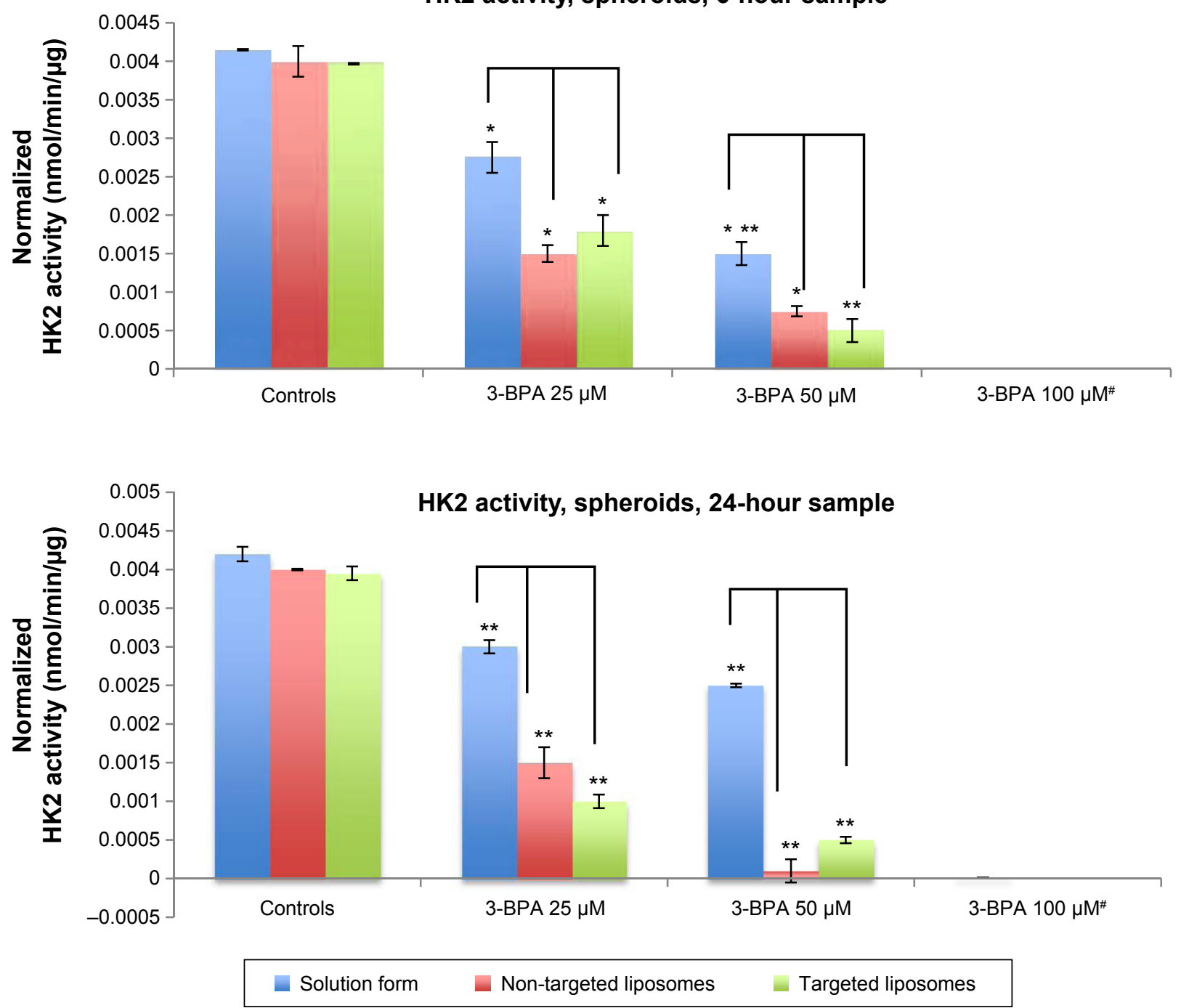

Figure 6 Evaluation of HK2 inhibition by 3-BPA delivered into SKOV-3 ovarian cancer cell spheroids using aqueous solution and non-targeted and targeted liposomal formulations at 3,6 , and 24 hours after treatment.

Notes: The values are reported as the mean \pm standard deviation for $n=3$. \#HK2 activity levels were below the detection limits of the assay for the I00 $\mu M$ samples. $* P<0.0$ I, $* * P<0.000$ I, Student's $t$-test (unpaired and two-tailed).

Abbreviations: 3-BPA, 3-bromopyruvate; HK2, hexokinase-2; min, minutes. 
Cytotoxicity of 3-BPA administered with control liposomes and EGFR-targeted liposomes

Comparison of the cytotoxic activity (Figure 7) of the liposomal formulations versus the solution form at various
3-BPA concentrations, ie, $100 \mu \mathrm{M}, 50 \mu \mathrm{M}, 25 \mu \mathrm{M}$, and $10 \mu \mathrm{M}$, shows that the liposomal formulations had a more potent cytotoxic effect than the solution form. The cytotoxic effect was more evident at lower concentrations $(10 \mu \mathrm{M}$ and $25 \mu \mathrm{M}$ ). Spheroids were more resistant to the drug,
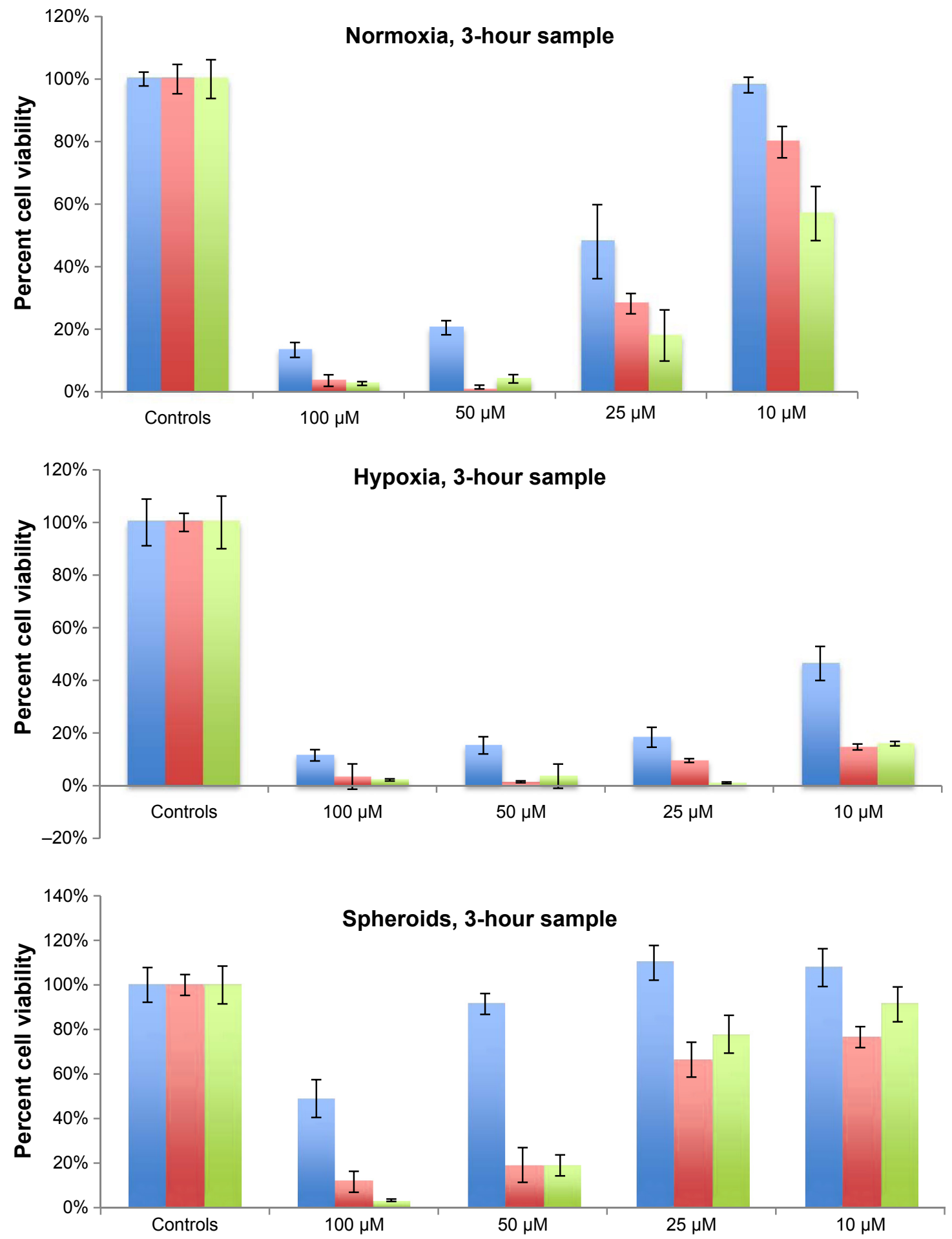

Solution form

Non-targeted liposomes

Targeted liposomes 

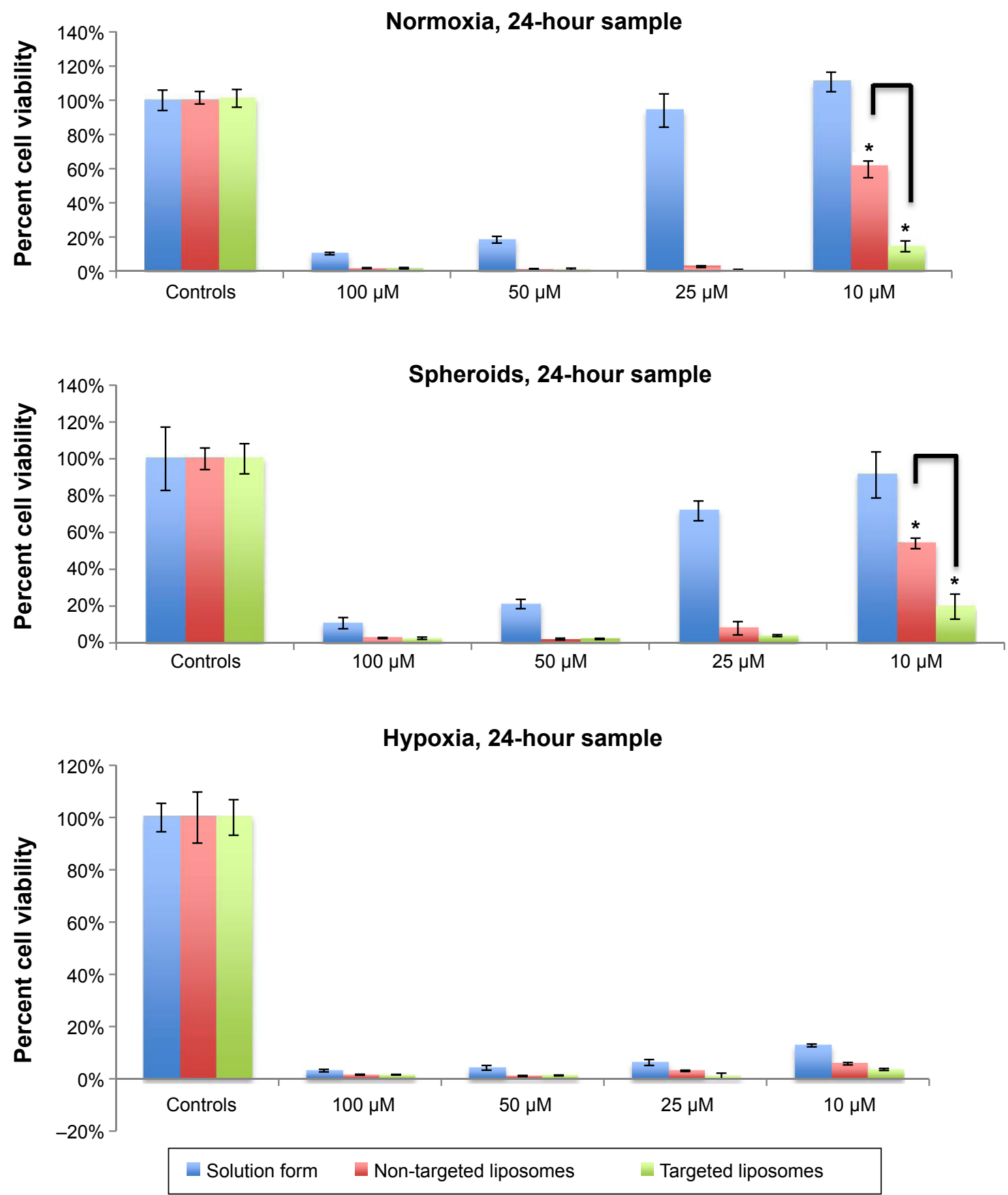

Figure 7 Evaluation of the cytotoxicity of 3-bromopyruvate delivered in SKOV-3 ovarian cancer cell spheroids using aqueous solution, and non-targeted and targeted liposomal formulations at 3 hours and 24 hours after treatment.

Notes: The values reported are the mean and standard deviation for $\mathrm{n}=3$. $* \mathrm{P}<0.000 \mathrm{I}$, Student's $t$-test (unpaired and two-tailed).

especially at lower concentrations, when compared with normoxic or hypoxic cells at the 3-hour time point. This observation can be explained by the amount of time taken by the drug in formulation or solution form to permeate all regions of the spheroids and show its cytotoxic effect. The targeted formulations had a better cytotoxic effect than the non-targeted formulations, especially the $10 \mu \mathrm{M}$ concentration at the 24-hour time point $(P<0.0001$, Student's $t$-test, unpaired and two-tailed).

\section{Discussion}

In the present study, we investigated the use of SKOV-3 cells for generating in vitro 3D tumor spheroids. Currently, there is a variety of methods, including forced floating, scaffolds, microfluidic cell culture platforms, liquid overlay, and hanging drop methods, that allow growth of cells as spheroids. ${ }^{8}$ In order to generate a 3D tumor spheroid model of SKOV-3 cells, we used the hanging drop method to grow spheroids reproducibly in a 96-well plate, which is suitable for high 
throughput screening of anticancer therapies. In order to test whether the ovarian cancer cells would grow in such a 3D arrangement, leading to recapitulation of the glycolytic modulation observed in the tumor microenvironment, we evaluated a panel of glycolytic enzymes (GLUT1, HK2, PKM2, and LDHA) which are overexpressed in animal models of ovarian, breast, or pancreatic cancer. ${ }^{4}$ The time points for evaluating the expression of these enzymes, ie, days 3 and 5, were chosen based on the fact that this time period allows for formation of intact spheroids/cell aggregates and also for buildup of hypoxia in the central region of the spheroids. From the gel images and densitometric analysis (Figure S3), we confirmed that these glycolytic markers, especially GLUT1, which is highly expressed and is a marker for increased glucose uptake, is expressed in hypoxic conditions and in spheroids after 3 and 5 days of initiation of spheroid formation. High expression levels of glycolytic markers also demonstrates that a glycolytic phenotype emerges in hypoxic conditions, which has been seen in other cancers, included esophageal, gastric, breast, and colon cancers. ${ }^{25}$

To further examine the difference in glycolytic marker levels in normoxic and spheroid growth conditions and to quantitatively determine expression at the enzyme/protein level, we used the hexokinase assay, which measures activity levels in given samples. Hexokinase activity was found to be upregulated in hypoxic cells from the day 3 and day 5 samples, which is in agreement with the fact that hypoxia-inducible factor- $1 \alpha$ upregulates downstream glycolytic enzymes. ${ }^{25}$ Interestingly, hexokinase activity levels in the spheroids were upregulated when compared with normoxic cells, but only in the day 5 samples. This could be attributed to the time required for buildup of hypoxia in spheroids. However, hypoxia is not the only factor needed for emergence of the glycolytic phenotype in cancer, and other pressures, such as oncogenic signals via the phosphatidylinositol 3-kinase/AKT/ mammalian target of rapamycin pathway, lead to elevated glycolytic activity, and mutations in mitochondrial DNA, which lead to loss of respiratory function in the mitochondria, drive cancer cells toward elevated glycolytic activity.,26

Unlike monolayer cell cultures, spheroids are 3D structures, and mass transport across these structures is by diffusion only, as in vivo tumors. Numerous mathematical models have been developed to predict the transport of drugs across the spheroid, and all lead to the conclusion that drug diffusion is the limiting parameter for drug efficacy. ${ }^{27,28}$ From a formulation and drug delivery stand point, this observation is crucial because the efficacy of a delivery system can be enhanced by modifying its physicochemical properties, such as surface charge or size, so as to achieve better penetration and efficacy in vivo. ${ }^{29}$ Using confocal microscopy, we demonstrated that liposomal formulations encapsulating rhodamine-dextran, a hydrophilic dye with a fluorescent signal, enhanced permeability to all regions of the spheroid. This became more evident from Z-stacking analysis of confocal images of the spheroid (Figure 4A-C), where the rhodamine signal was observed not only at the periphery of the spheroid but also in its central region. The enhanced permeability can be attributed to the ability of the liposomal formulations to permeate across the interstitial spaces in the spheroids, and this was achieved by incorporation of fusogenic lipids like DSPC and DSPE-PEG into the lipid bilayer during their preparation. ${ }^{30}$

Lastly, in order to investigate whether 3D arrangement of cells physically prevents drug penetration and whether upregulation of glycolytic activity in spheroids leads to further resistance to 3-BPA in terms of hexokinase inhibition and cytotoxicity, we used 3-BPA as an inhibitor of aerobic glycolysis in spheroids. Cells grown in spheroid cultures exhibited greater resistance in comparison with monolayer cell cultures when treated with 3-BPA in both solution and liposomal formulations. Both the hexokinase inhibition study and the cytotoxicity study (Figures 6 and 7) demonstrated a dose-dependent effect, with $100 \mu \mathrm{M}$ being the most potent and achieving $100 \%$ cytotoxicity at both time points, ie, 3 hours and 24 hours, with the $10 \mu \mathrm{M}$ dose being the least potent. However, there was not much difference between the EGFR-targeted and non-targeted liposomal formulations in terms of hexokinase inhibition and cytotoxic effects. The reason for this could be that the amount of surface modification of the liposomal formulation with the EGFR peptide could not demonstrate the differential uptake between targeted and non-targeted formulations, which in turn might not have translated into inhibition of hexokinase and the cytotoxic effects of the targeted formulation. Nevertheless, the liposomal formulations enhanced the overall efficacy of 3-BPA, with an at least 2-5 fold increase in hexokinase inhibition and cytotoxic effects at the 24-hour time point for the various concentrations, and this can be attributed to the greater penetration demonstrated by the permeability study and uptake at all concentrations of 3-BPA when compared with its solution form.

\section{Conclusion}

SKOV-3 spheroids can be reproducibly developed using a 96-well hanging drop method. HK2 enzyme activity levels in hypoxic conditions (including growth in spheroids) were 
found to be higher than in normoxic conditions. The permeability studies carried out using fluorescence confocal microscopy showed better penetration of rhodamine-dextranencapsulated liposomes into the spheroids when compared with free rhodamine-dextran dye in solution. The liposomal formulations had a better HK2 inhibitory and cytotoxic effects in spheroids when compared with the solution form of 3-BPA. Our data support the usefulness of ovarian cancer cell spheroids as a model for tumor aerobic glycolysis, and the biological assays used, such as hexokinase, and cytotoxicity together confirm the enhanced potency of 3-BPA when delivered in liposomal form.

\section{Acknowledgments}

This study was supported by a Cancer Nanotechnology Platform Partnership grant (U01-CA151452) from the National Cancer Institute's Alliance in Nanotechnology and by the Northeastern University/Dana Farber Cancer Institute's Development of Cancer Therapeutics Program. The authors appreciate the help provided by Huyen Tran (Northeastern University, Boston, MA, USA) in confocal imaging of the spheroid samples.

\section{Disclosure}

The authors report no conflicts of interest in this work.

\section{References}

1. American Cancer Society. Cancer Facts and Figures 2014. Available from: http://www.cancer.org/research/cancerfactsstatistics/ cancerfactsfigures2014/. Accessed May 29, 2015.

2. Guarneri V, Piacentini F, Barbieri E, Conte PF. Achievements and unmet needs in the management of advanced ovarian cancer. Gynecol Oncol. 2010;117(2):152-158.

3. Gillies RJ, Robey I, Gatenby RA. Causes and consequences of increased glucose metabolism of cancers. J Nucl Med. 2008;49 Suppl 2: 24S-42S.

4. Pelicano H, Martin DS, Xu RH, Huang P. Glycolysis inhibition for anticancer treatment. Oncogene. 2006;25(34):4633-4646.

5. Fennema E, Rivron N, Rouwkema J, van Blitterswijk C, de Boer J. Spheroid culture as a tool for creating 3D complex tissues. Trends Biotechnol. 2013;31(2):108-115.

6. Chang TT, Hughes-Fulford M. Monolayer and spheroid culture of human liver hepatocellular carcinoma cell line cells demonstrate distinct global gene expression patterns and functional phenotypes. Tissue Eng Part A. 2009;15(3):559-567.

7. Knuchel R, Hofstadter F, Jenkins WE, Masters JR. Sensitivities of monolayers and spheroids of the human bladder cancer cell line MGH-U1 to the drugs used for intravesical chemotherapy. Cancer Res. 1989; 49(6):1397-1401.

8. Haycock JW. 3D cell culture: a review of current approaches and techniques. Methods Mol Biol. 2011;695:1-15.

9. Friedrich J, Seidel C, Ebner R, Kunz-Schughart LA. Spheroid-based drug screen: considerations and practical approach. Nat Protoc. 2009;4(3): 309-324.
10. Mueller-Klieser W, Freyer JP, Sutherland RM. Influence of glucose and oxygen supply conditions on the oxygenation of multicellular spheroids. Br J Cancer. 1986;53(3):345-353.

11. Ko YH, Verhoeven HA, Lee MJ, Corbin DJ, Vogl TJ, Pedersen PL. A translational study "case report" on the small molecule "energy blocker" 3-bromopyruvate (3BP) as a potent anticancer agent: from bench side to bedside. J Bioenerg Biomembr. 2012;44(1):163-170.

12. Pereira da Silva AP, El-Bacha T, Kyaw N, et al. Inhibition of energyproducing pathways of HepG2 cells by 3-bromopyruvate. Biochem J. 2009;417(3):717-726.

13. Ko YH, Smith BL, Wang Y, et al. Advanced cancers: eradication in all cases using 3-bromopyruvate therapy to deplete ATP. Biochem Biophys Res Commun. 2004;324(1):269-275.

14. Ko YH, Pedersen PL, Geschwind JF. Glucose catabolism in the rabbit VX2 tumor model for liver cancer: characterization and targeting hexokinase. Cancer Lett. 2001;173(1):83-91.

15. Mathupala SP, Rempel A, Pedersen PL. Glucose catabolism in cancer cells identification and characterization of a marked activation response of the type II hexokinase gene to hypoxic conditions. $J$ Biol Chem. 2001;276(46):43407-43412.

16. Patra KC, Wang Q, Bhaskar PT, et al. Hexokinase 2 is required for tumor initiation and maintenance and its systemic deletion is therapeutic in mouse models of cancer. Cancer Cell. 2013;24(2):213-228.

17. Shoshan MC. 3-Bromopyruvate: targets and outcomes. J Bioenerg Biomembr. 2012;44(1):7-15.

18. Bangham AD. Liposomes: the Babraham connection. Chem Phys Lipids. 1993;64(1-3):275-285.

19. Alexis F, Pridgen EM, Langer R, Farokhzad OC. Nanoparticle technologies for cancer therapy. In: Schäfer-Korting M, editor. Drug Delivery. Heidelberg, Germany: Springer; 2010.

20. Ganta S, Devalapally H, Shahiwala A, Amiji M. A review of stimuliresponsive nanocarriers for drug and gene delivery. J Control Release. 2008;126(3):187-204.

21. Xu X, Khan MA, Burgess DJ. A quality by design (QbD) case study on liposomes containing hydrophilic API: II. Screening of critical variables, and establishment of design space at laboratory scale. Int $J$ Pharm. 2012;423(2):543-553.

22. Magadala P, Amiji M. Epidermal growth factor receptor-targeted gelatin-based engineered nanocarriers for DNA delivery and transfection in human pancreatic cancer cells. AAPS J. 2008;10(4):565-576.

23. Mendelsohn J, Baselga J. Epidermal growth factor receptor targeting in cancer. Semin Oncol. 2006;33(4):369-385.

24. Li Z, Zhao R, Wu X, et al. Identification and characterization of a novel peptide ligand of epidermal growth factor receptor for targeted delivery of therapeutics. FASEB J. 2005;19(14):1978-1985.

25. Gatenby RA, Gillies RJ. Why do cancers have high aerobic glycolysis? Nat Rev Cancer. 2004;4(11):891-899.

26. Ishikawa $\mathrm{K}$, Takenaga $\mathrm{K}$, Akimoto $\mathrm{M}$, et al. ROS-generating mitochondrial DNA mutations can regulate tumor cell metastasis. Science. 2008;320(5876):661-664.

27. Ward JP, King JR. Mathematical modelling of drug transport in tumour multicell spheroids and monolayer cultures. Math Biosci. 2003;181(2):177-207.

28. Wenning LA, Murphy RM. Coupled cellular trafficking and diffusional limitations in delivery of immunotoxins to multicell tumor spheroids. Biotechnol Bioeng. 1999;62(5):562-575.

29. Kostarelos K, Emfietzoglou D, Papakostas A, Yang WH, Ballangrud AM, Sgouros G. Engineering lipid vesicles of enhanced intratumoral transport capabilities: correlating liposome characteristics with penetration into human prostate tumor spheroids. J Liposome Res. 2005;15(1-2):15-27.

30. Kostarelos K, Emfietzoglou D, Papakostas A, Yang WH, Ballangrud A, Sgouros G. Binding and interstitial penetration of liposomes within avascular tumor spheroids. Int J Cancer. 2004;112(4):713-721. 


\section{Supplementary materials}

\section{Synthesis of lipid-EGFR peptide conjugate}

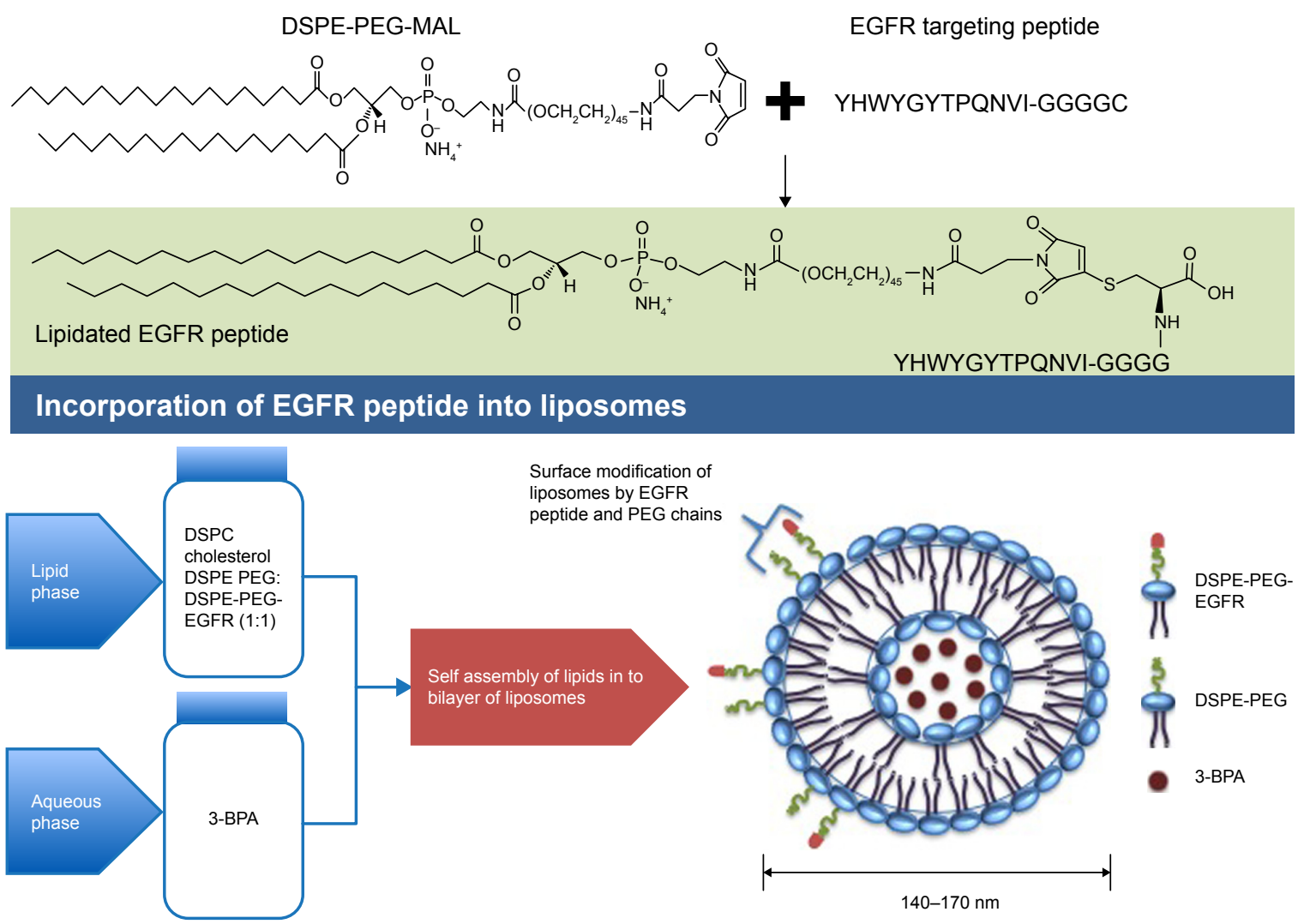

Figure SI Synthesis of EGFR peptide-modified DSPE-PEG-MAL and its incorporation into liposomes.

Abbreviations: 3-BPA, 3-bromopyruvate; DSPC, I,2-distearoyl-sn-glycero-3-phosphocholine; DSPE, I,2-distearoyl-sn-glycero-3-phosphoethanolamine; EGFR, epidermal growth factor receptor; MAL, maleimide; PEG, polyethylene glycol. 
A

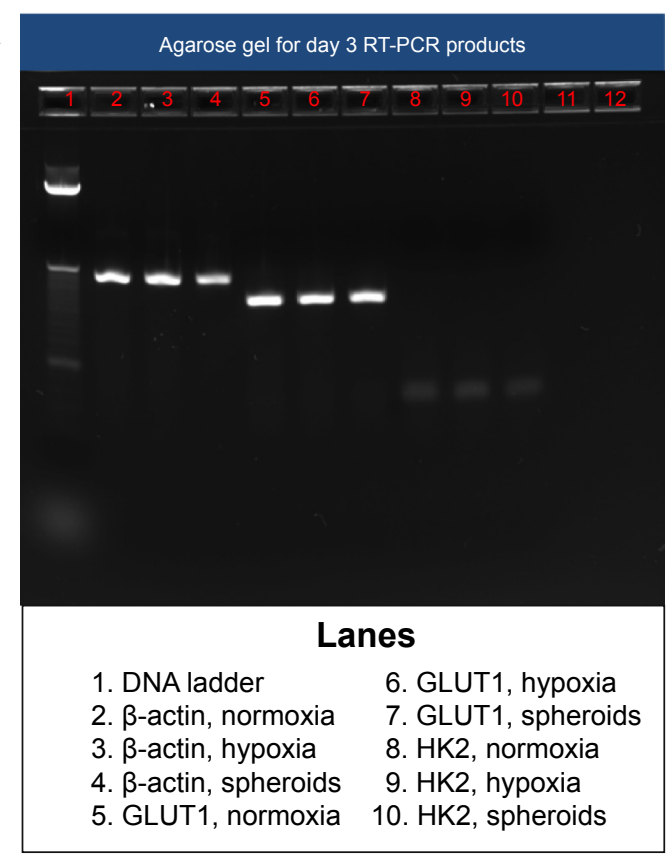

C

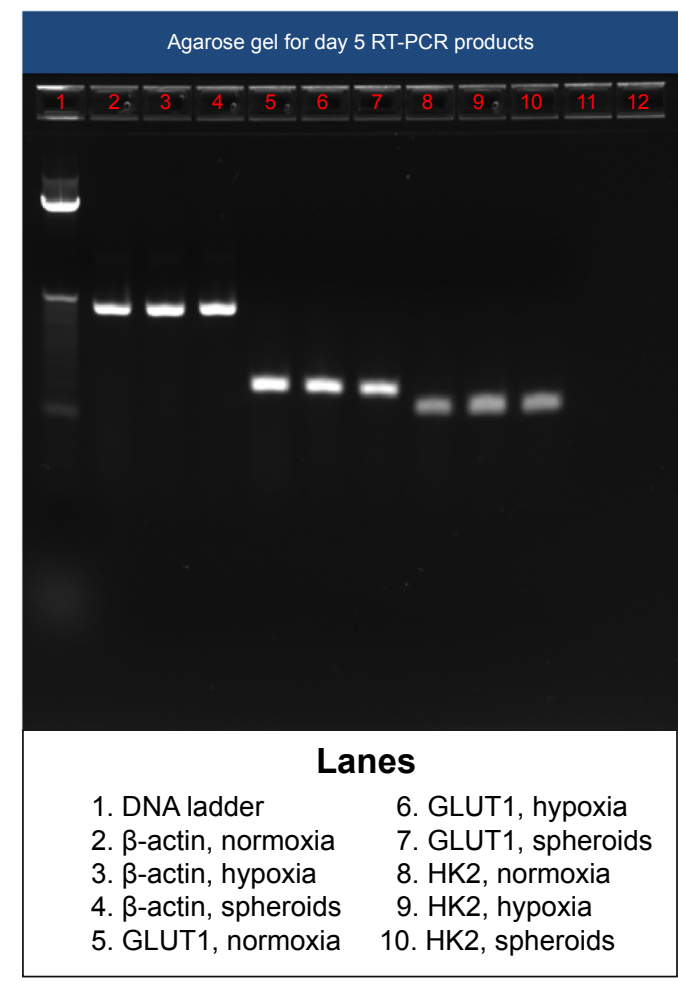

B

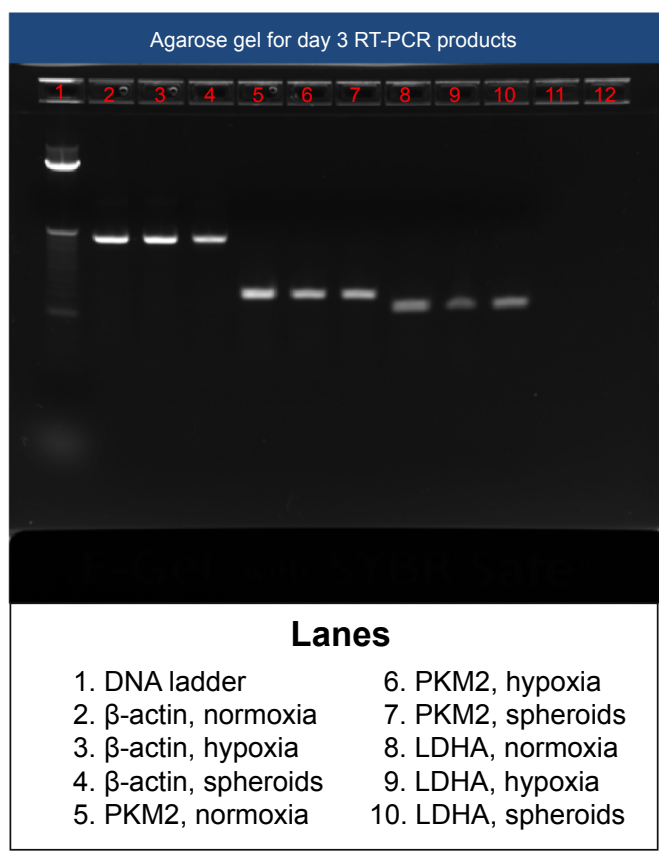

D

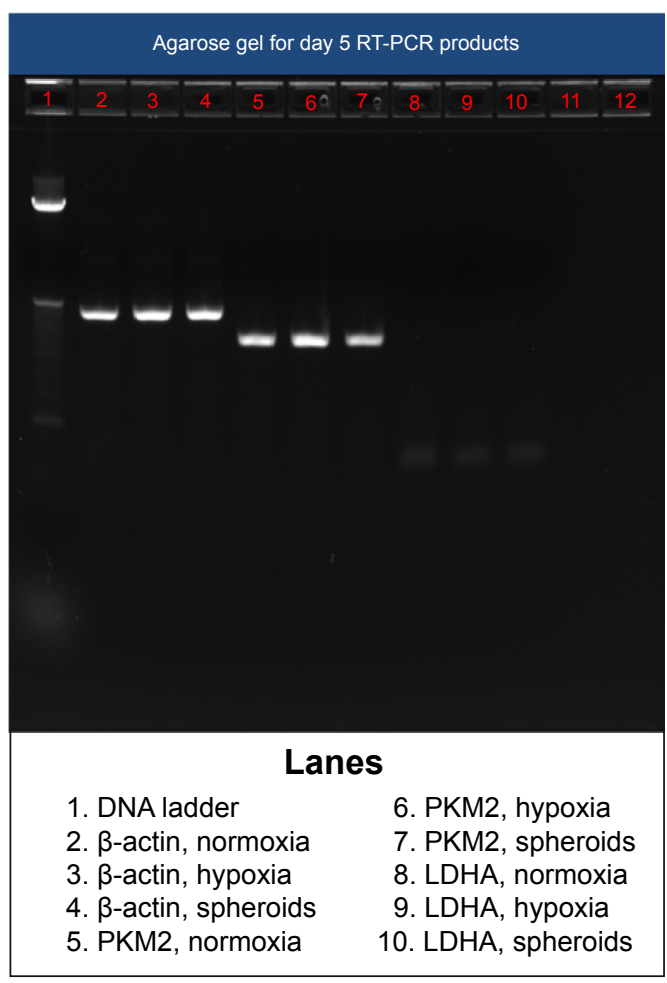

Figure S2 RT-PCR analysis of glycolytic markers in two-dimensional SKOV-3 ovarian cancer cell culture and in three-dimensional spheroids.

Notes: The bands run on the 2\% agarose E-gel image show relative expression of (A) GLUT I and HK2 for day 3 samples, (B) PKM2 and LDHA for day 3 samples, (C) GLUT I and HK2 for day 5 samples, and (D) PKM2 and LDHA for day 5 samples. Lanes II and I2 in both the gels are blank lanes run with deionized water.

Abbreviations: GLUTI, glucose transporter I; HK2, hexokinase-2; LDHA, lactate dehydrogenase A; PKM2, pyruvate kinase isoform M2; RT-PCR, reverse transcription polymerase chain reaction. 

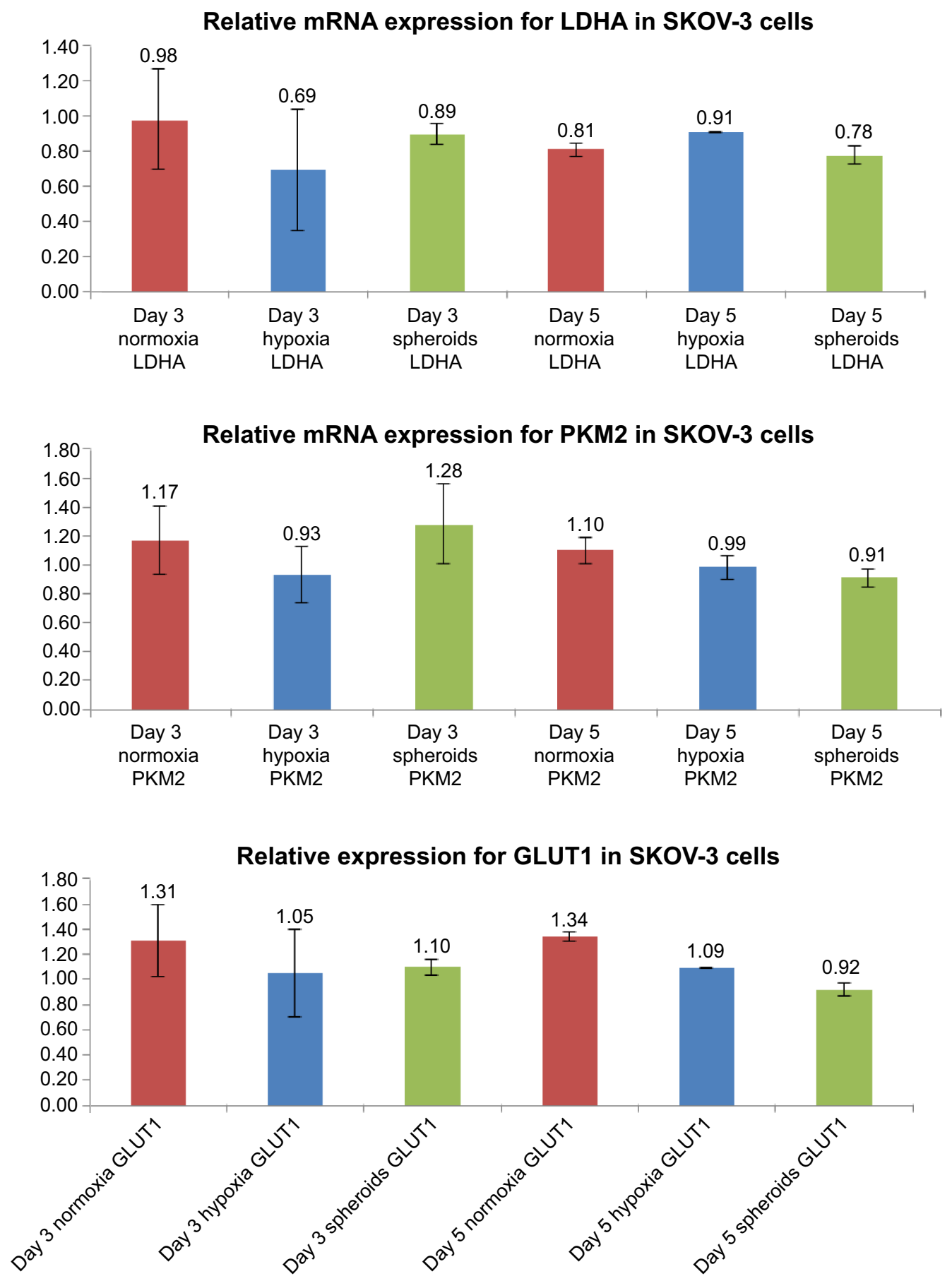

Figure S3 Comparative analysis of glycolytic marker expression profiles in a two-dimensional SKOV-3 ovarian cancer cell culture and in three-dimensional spheroids. Notes: mRNA expression levels of each gene were normalized to the respective $\beta$-actin band intensity. The values are reported as the mean \pm standard deviation calculated for $n=3$. The samples correspond to day 3 normoxic samples, day 5 normoxic samples, day 3 hypoxic samples, and day 5 hypoxic samples.

Abbreviations: GLUTI, glucose transporter I; LDHA, lactate dehydrogenase A; PKM2, pyruvate kinase isoform M2. 

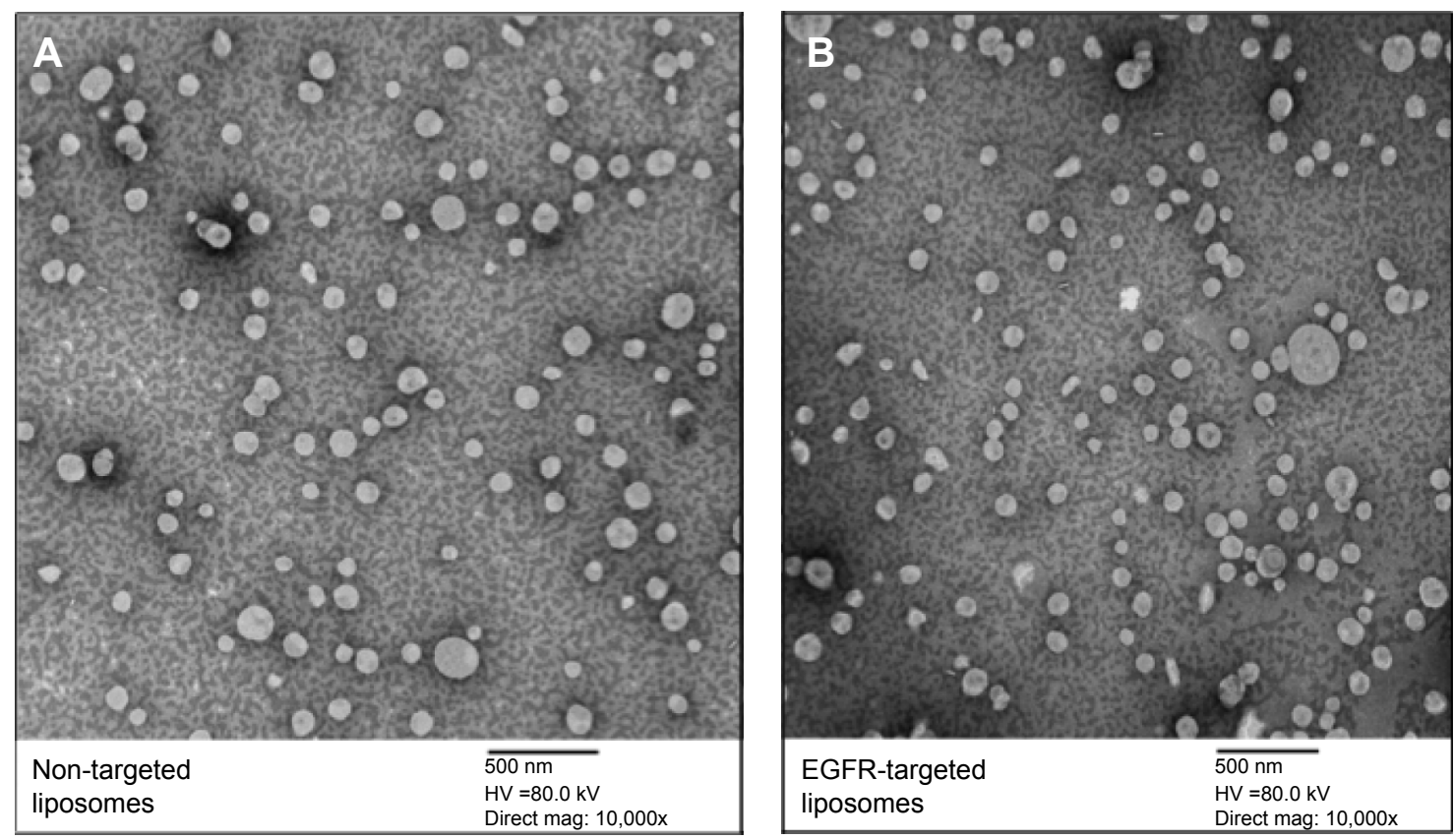

Figure S4 Transmission electron microscopic images of 3-bromopyruvate-loaded liposomes revealing spherical morphology and size.

Notes: (A) Non-targeted liposomes and (B) EGFR-targeted liposomes. Original magnification of the transmission electron micrographs was I0,000× with a scale bar of $500 \mathrm{~nm}$.

Abbreviations: EGFR, epidermal growth factor receptor; HV, high voltage; mag, magnification.

International Journal of Nanomedicine

\section{Publish your work in this journal}

The International Journal of Nanomedicine is an international, peerreviewed journal focusing on the application of nanotechnology in diagnostics, therapeutics, and drug delivery systems throughou the biomedical field. This journal is indexed on PubMed Central, MedLine, CAS, SciSearch ${ }^{\circledR}$, Current Contents ${ }^{\circledR} /$ Clinical Medicine,

\section{Dovepress}

Journal Citation Reports/Science Edition, EMBase, Scopus and the Elsevier Bibliographic databases. The manuscript management system is completely online and includes a very quick and fair peer-review system, which is all easy to use. Visit http://www.dovepress.com/ testimonials.php to read real quotes from published authors.

\footnotetext{
Submit your manuscript here: http://www.dovepress.com/international-journal-of-nanomedicine-journal
} 